\title{
Multicarrier code for the next-generation GPS
}

\author{
Donglin Wang ${ }^{1,2^{*}}$, Michel Fattouche ${ }^{1}$ and Fadhel M Ghannouchi ${ }^{1}$
}

\begin{abstract}
This article investigates multicarrier (MC) transmission for next-generation global positioning system (GPS) instead of current spread spectrum signals. A MC code is proposed in this article as an alternative to the coarse/acquisition (C/A) code in GPS. The entire GPS bandwidth for the C/A code is divided into 1,024 subcarrier slots. As per our proposed arrangement, each satellite vehicle (SV) takes up only 42 uniformly spaced and non-overlapping subcarrier slots while approximately occupying the same bandwidth as the C/A code in GPS. In this way, the proposed MC code is proved to attain a $4.73 \mathrm{~dB}$ SNR gain compared to the GPS C/A code in terms of Cramer-Rao lower bound for range estimation, which could evidently enhance the GPS receiver's sensitivity. Together with the feature of robustness against multipath effect, the proposed MC code is helpful for urban, tunnel, even indoor and underground positioning. The transmission and reception of the propose MC code is also described, where the range estimation process is explained. Furthermore, the proposed MC code is shown to be robust against narrow band interference. Moreover, the probability of collision between SVs due to Doppler shifts is theoretically analyzed, where the probability of successful positioning is evaluated. Simulation Results show a consistency with our proposed theory.
\end{abstract}

Keywords: multicarrier code, GPS C/A code, SNR Gain, doppler effect, NBI, probability of collision

\section{Introduction}

Global navigation satellite system (GNSS) such as the current global positioning system (GPS) or the future European Galileo system can provide a worldwide accurate positioning under an outdoor good environmental conditions [1]. However, GNSS based positioning is not reliable in urban, tunnel, underbridge, indoor or underground environments due to the dense multipath effect as well as non-line-of-sight (NLOS) signal energy attenuation [1]. As it is known, multicarrier (MC) signals, e.g. orthogonal frequency division multiplexing (OFDM) signals $[2,3]$, are robust against the multipath effect [4-8]. Furthermore, they have a more efficient spectrum usage compared to the current golden codes $[9,10]$, which thus leads to a SNR gain for range estimation. Therefore, the MC signal is being considered for investigation of the possiblity of its application in next-generation GNSS.

For the best of authors' knowledge, the use of a MC signal as a code of next-generation GNSS is investigated by both [1,11]. Zanier and Luise [11] discussed about the funamental issues in time-delay estimation of MC

\footnotetext{
* Correspondence: dowang@ucalgary.ca

'ECE Department, University of Calgary, Calgary, AB, T2N 1N4, Canada Full list of author information is available at the end of the article
}

signals with applications to next-generation GNSS. This article used a filter bank MC modulation, where the MC modulation has the same power spectral density (PSD) of the current golden code so that they have the same ranging performance. Furthermore, Zanier and Luise [11] derived the fundamental performance of the filter bank MC modulation using Cramer-Rao lower bound (CRLB). Dai et al. [1] tried to propose the OFDM/MC based scheme for the next-generation GNSS. However, authors basically discussed the OFDM communication and the performance of time-of-arrival (TOA) estimation, which are too far from an available GNSS scheme. As we know, it is impossible for all SVs to use the same OFDM signal when the frequency collision between SVs will occur and the data reception will fail without question. Dai et al. [1] only gave a general discussion but did not provide the specific probing signal for each satellite vehicle (SV). Also, it did not compare the OFDM signal with the current GNSS code in terms of ranging and/or positioning accuracy. Furthermore, it did not analyze the scheme's feasibility as an option of the next-generation GNSS.

Differently from the previous literature, this article investigates the possiblity of a unfiltered non-data-bearing MC modulation applied in the next-generation 
GNSS instead of the current golden code. Specifically, a $\mathrm{MC}$ code is proposed as an alternative to the coarse/ acquisition ( $\mathrm{C} / \mathrm{A}$ ) code for the next-generation GPS. As we all know, GPS transmits three binary codes for target navigation: the pseudo-random noise $(\mathrm{PN})$ based $\mathrm{C} / \mathrm{A}$ code with $1.023 \mathrm{MHz}$, the PN-based precise $(P)$ code with $10.23 \mathrm{MHz}$ and the navigation message with 50 bps [12-15]. Among them, the C/A code is for civilian use but performs poor under the dense multipath effect, e.g. positioning in a downtown environment, weak signal detection such as indoor or underground positioning and narrow band interference (NBI). ${ }^{a}$ Consequently, the $\mathrm{MC}$ modulation is proposed as a next-generation alternative to the $\mathrm{C} / \mathrm{A}$ code in order to improve the positioning performance against multipath, weak signal and NBI. Even though the multipath management in the communication area is different from that in the GNSS area, the topic of the multipath management is not addressed in the article.

In our proposed design, the entire GPS bandwidth for the C/A code, i.e. a null-to-null bandwidth $2.048 \mathrm{MHz}$, ${ }^{\mathrm{b}}$ is divided into 1,024 subcarrier slots. Under the assumption ${ }^{\mathrm{C}}$ that there are currently 24 SVs in GPS, each SV takes up only 42 uniformly spaced and non-overlapping subcarrier slots as per our proposed arrangement while approximately occupying the whole bandwidth. In this way, ${ }^{\mathrm{d}}$ the proposed MC code is proved to attain a $4.73 \mathrm{~dB}$ improvement (See Section 3) to that of the C/A code in terms of ranging accuracy. In other words, for a fixed ranging accuracy, the required SNR is $4.73 \mathrm{~dB}$ lower than that for the
C/A code, indicating a $4.73 \mathrm{~dB}$ SNR gain when using the proposed $\mathrm{MC}$ codes instead of the current spread spectrum signals. The proposed MC code is also proven to be robust against NBI. Besides, the effect of Doppler shift to positioning process is proved to be negligible.

The remainder of the article is organized as follows. Section 2 describes the proposed MC code for all 24 SVs in GPS. Section 3 proves that the proposed MC code attains a $4.73 \mathrm{~dB}$ SNR gain compared to the current C/A code. The transmission and reception of the proposed $\mathrm{MC}$ code is described in Section 4, followed by the analysis of NBI effect on the proposed MC code in Section 5. Section 6 thoroughly analyzes the upper bound of Doppler effect on the reception of the proposed probing signals, by defining the probability of collision (POC) and the probability of successful positioning. Simulation results are given in Section 7, followed by conclusion in Section 8.

\section{Proposed MC multiple access method for each SV}

We propose to divide the entire $2.048 \mathrm{MHz}$ band into 1,024 non-overlapping orthogonal subcarrier slots as shown in Figure 1, where each subcarrier slot has a null-to-null bandwidth of approximately $2 \mathrm{kHz}$. In our proposed design, the first and last eight subcarrier slots are left blank while the remaining 1,008 subcarrier slots are used to transmit the probing signals, namely, 42 unique subcarrier slots per SV. Due to the facts that (i) occupying the whole bandwidth results in a good

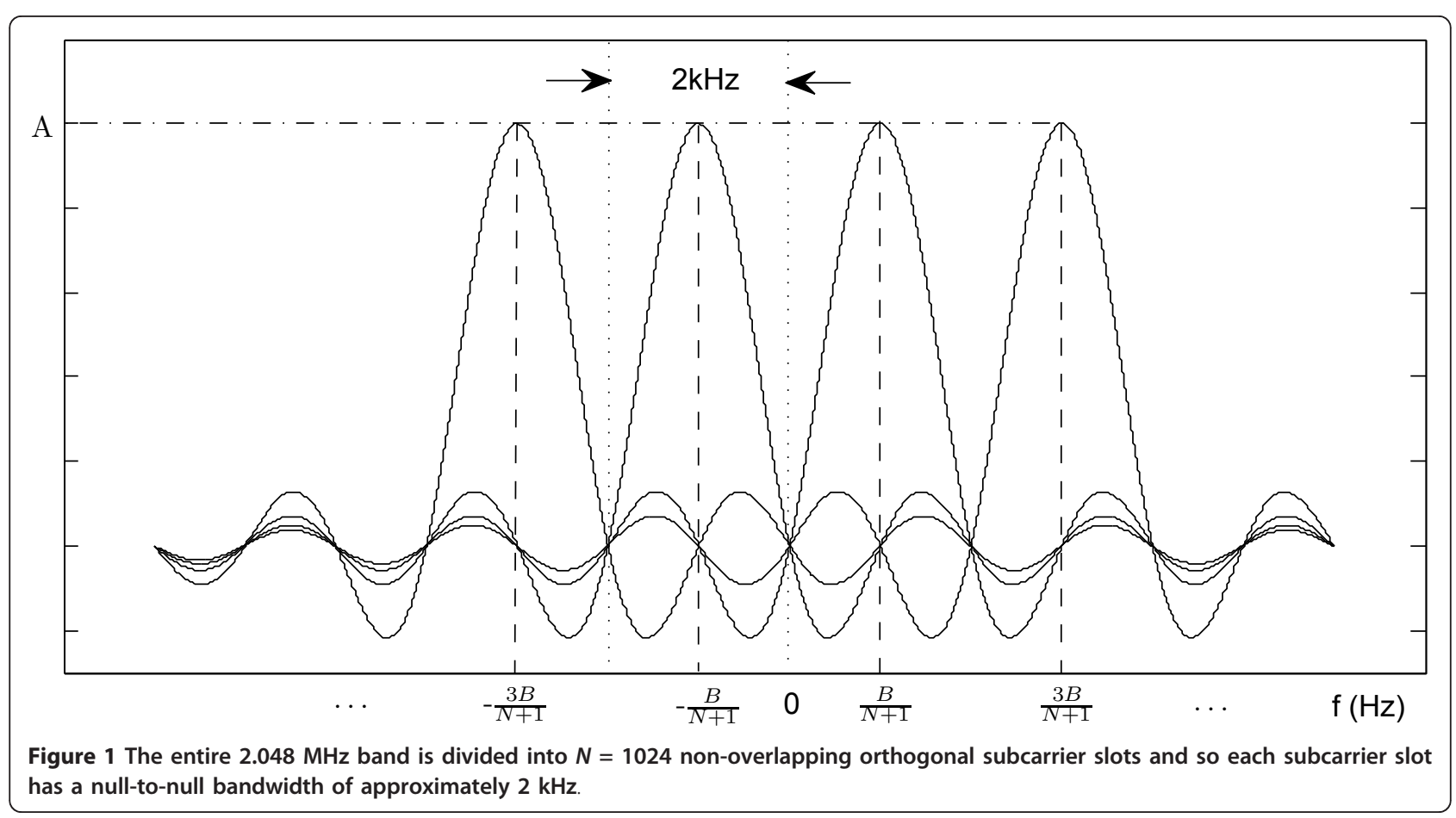


ranging accuracy [16] and (ii) it is generally necessary to have all 24 SVs in GPS with more or less the same ranging accuracy since 4 to $12 \mathrm{SVs}$ are usually visible at any point on earth and at any time, each group of 42 non-overlapping subcarrier slots is selected uniformly to cover the entire $2.048 \mathrm{MHz}$ band.

Define $S_{I D}$ as a number from 1 to 24 that is used to identify each SV. Define $I_{D}$ as an index set which is used to identify the subcarrier slots that are allocated to each SV. According to our design, an SV with the number $S_{I D}$, is allocated the index set, $I_{D}$, as

$$
I_{D}=8+\left(S_{I D}-1\right)+\left(S_{\text {index }}-1\right) \times 24,
$$

where $S_{\text {index }}$ is an integer from 1 to 42 , which denotes the specific subcarrier slot allocated to the $S_{\mathrm{ID}}^{\text {th }} \mathrm{SV}$. Define $S(f)$ as the continuous expression for the MC code in the frequency domain. It, in turn, can be sampled at $f= \pm \frac{B}{N+1}, \pm \frac{3 B}{N+1}, \ldots, \pm \frac{(N-1) B}{N+1}, N=1024$ and $B=1.023 \mathrm{MHz}$, which is shown in Figure 1 as dash lines. The resulting discrete expression is given by $S[k]=S\left(\frac{(2 k-N+1) B}{N+1}\right)$ for $k=0,1, \ldots, N-1$. More specifically, for the $S_{\mathrm{ID}}^{\text {th }} \mathrm{SV}$, we have

$$
S[k]= \begin{cases}A, & k \in I_{D} \\ 0, & \text { others }\end{cases}
$$

where $A$ is a constant as shown in Figure 1 and the value of $A$ is determined by the transmitter power.

Equation (1) is explained further in Figure 2a, where 1,024 subcarrier slots are divided into 42 groups. Each group contains 24 subcarrier slots and the first and last eight subcarrier slots are left blank. Specifically, subcarrier slots allocated to the $k$ th, SV, are shown in Figure $2 \mathrm{~b}$, which includes the $k$ th subcarrier of each group.

\section{Ranging accuracy and SNR gain}

The CRLB for time-based range estimation, $\hat{R}$, can be expressed as $[9,16]$

$$
\operatorname{Var}\{\hat{R}\} \geq \frac{c^{2}}{\frac{|a|^{2} \xi}{N_{0} / 2} \mathscr{B}^{2}},
$$

where $\xi=\sum_{k=0}^{N-1}|S[k]|^{2}$ is the discrete transmitted energy; $a$ denotes the complex-valued attenuation of the direct path; $|\cdot|$ denotes modulus; $\frac{N_{0}}{2}$ is the PSD of the additional white Gaussian noise (AWGN) in the propagation channel; $\frac{|a|^{2} \xi}{N_{0} / 2}$ is the received SNR; $c$ is the speed of light; $\mathscr{B}^{2}$ is the mean square bandwidth (MSB), which can be approximated in a discrete form as

$$
\mathscr{B}^{2} \stackrel{(a)}{\approx}\left(\frac{2 \pi B}{N+1}\right)^{2} \frac{\sum_{k=0}^{N-1}(2 k-N+1)^{2}|S[k]|^{2}}{\sum_{k=0}^{N-1}|S[k]|^{2}},
$$

where $B=1.023 \mathrm{MHz}$, and approximation ( $a$ ) occurs due to the transformation from a continuous form to a discrete form, which will be verified by simulation. The PSD for the PN-based C/A code in GPS is a sinc square function [17-19], which implies that, $S[k]$ in (4), is a sampled mainlobe of the sinc function. On the other hand, for our proposed design, the MSB can be further expressed as

$$
\begin{aligned}
\mathscr{B}^{2} & \stackrel{(a)}{\approx}\left(\frac{2 \pi B}{N+1}\right)^{2} \frac{\sum_{k \in I_{D}}(2 k-N+1)^{2} A^{2}}{\sum_{k \in I_{D}} A^{2}} \\
& =\left(\frac{2 \pi B}{1025}\right)^{2} \frac{\sum_{k \in I_{D}}(2 k-1023)^{2}}{42}
\end{aligned}
$$

where $I_{D}$ is given in (1).

Based on (5), the msb in $\mathrm{MHz}\left(m s b \triangleq \frac{\sqrt{\mathrm{MSB}}}{2 \pi} \mathrm{MHz}\right)$ for the proposed MC code is calculated and illustrated in Table 1 for all SVs, i.e. $S_{I D}=1,2, \ldots, 24$. From Table 1 , one can see that the proposed design has a MSB ranging from 0.5905 to $0.5909 \mathrm{MHz}$, implying that the 24 SVs have an approximately equal ranging accuracy. On the other hand, the MSB in MHz for the PN-based C/A code in GPS is obtained from (4) as $0.3427 \mathrm{MHz}$. One note to be pointed out is that the ID in Table 1 denotes the standard name in GPS and can be generally represented as $L m$, where $L$ is the orbit plane, from $A$ to $F$, and $m$ is the specific SV, from 1 to 4 [12]. The $S_{I D}$ defined in this article is matched to the standard ID and shown in Table 1.

Denote $\mathrm{MSB}_{s}, \mathrm{CRLB} s$ and $\left(\frac{\left|a_{0}\right|^{2} \xi}{N_{0} / 2}\right)_{s}$ as the MSB, the CRLB and the SNR for the proposed MC code, respectively. Similarly, denote $\operatorname{MSB}_{c}, \mathrm{CRLB}_{c}$ and $\left(\frac{\left|a_{0}\right|^{2} \xi}{N_{0} / 2}\right)_{c}$ as the MSB, the CRLB and the SNR for the PN-based C/A code, respectively. The difference between the proposed $\mathrm{MC}$ code and the C/A code, in terms of CRLB for range estimation under an equal SNR, can be obtained as

$$
\begin{aligned}
& \operatorname{CRLB}_{s}(\mathrm{~dB})-\mathrm{CRLB}_{c}(\mathrm{~dB}) \\
= & \operatorname{MSB}_{c}(\mathrm{~dB})-\mathrm{MSB}_{s}(\mathrm{~dB}) \stackrel{(a)}{\approx}-4.73(\mathrm{~dB}) .
\end{aligned}
$$

From another viewpoint, for a fixed ranging accuracy, the difference of the required SNR between the proposed design and the C/A code is obtained as

$$
\left(\frac{\left|a_{0}\right|^{2} \xi}{N_{0} / 2}\right)_{s}(\mathrm{~dB})-\left(\frac{\left|a_{0}\right|^{2} \xi}{N_{0} / 2}\right)_{c}(\mathrm{~dB}) \stackrel{(a)}{\approx}-4.73(\mathrm{~dB}),
$$




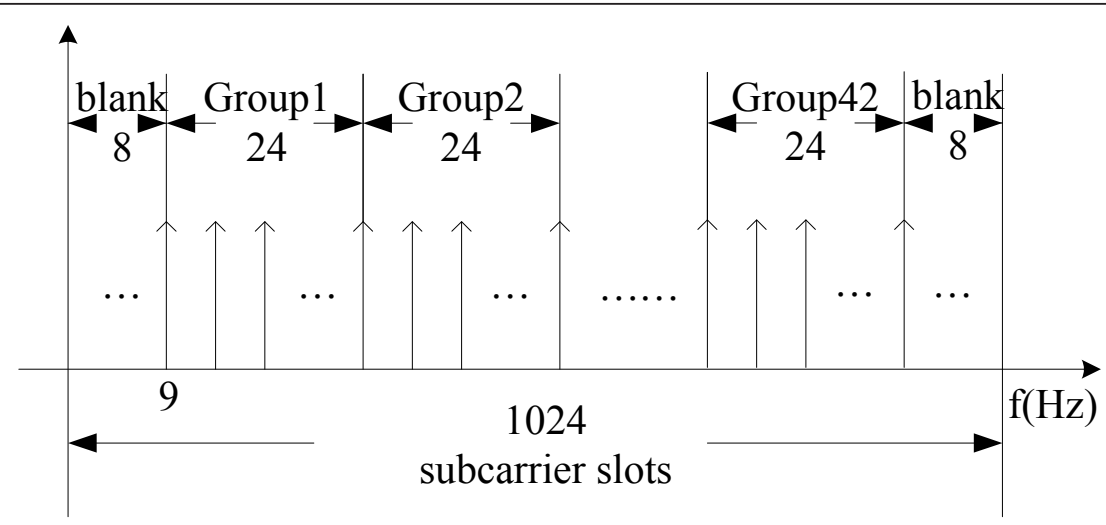

(a)

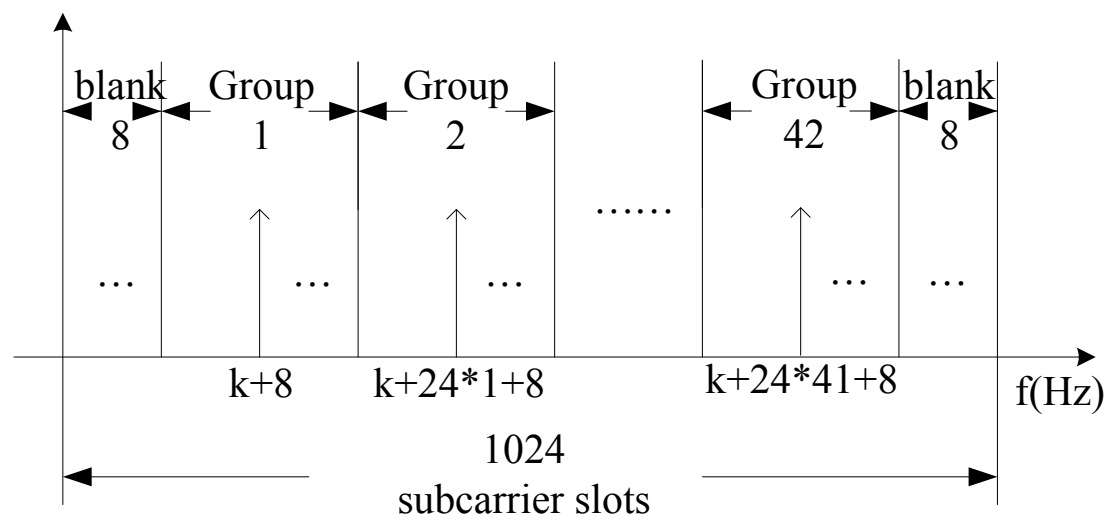

(b)

Figure 2 Proposed MC code for GPS: (a) The entire $2.048 \mathrm{MHz}$ band is uniformly divided into 1024 subcarrier slots, where the first and last 8 are left blank while the remaining 1008 are divided into 42 groups, each with 24 subcarriers. (b) 42 subcarriers are allocated to the $k^{\text {th }}$ SV, $k=1$, $2, \ldots, 24$, which is made up of the kth subcarrier slot in each group.

Which indicates that using the proposed $\mathrm{MC}$ code in GPS would lead to a $4.73 \mathrm{~dB}$ SNR gain compared to the current spread spectrum's gold code.

\section{Transmission and reception of the proposed MC code}

As known, the data rate of the navigation message in current GPS is 50 bps while the bandwidth used for the proposed MC code is $1.024 \mathrm{MHz}$. The navigation message is a telemetry message, and the data is transmitted in logical units called frames. For GPS, a frame is 1,500 bits long and takes $30 \mathrm{~s}$ to be transmitted. Each frame is divided into five subframes, 300 bits long per subframe. Subframes 1, 2 and 3 contain the high accuracy ephemeris and clock off set data. Subframes 4 and 5 contain the almanac data and some related health and configuration data [12].

As shown in Figure 3, the proposed MC code is firstly modulated by the navigation message. Furthermore, the

Table 1 MSB in MHz, i.e. $m s b \triangleq \frac{\sqrt{M S B}}{2 \pi} \mathrm{MHz}$, for our proposed MC code with $S_{I D}=1,2, \ldots, 24$, and the PN-based C/A code

\begin{tabular}{|c|c|c|c|c|c|c|c|c|c|c|c|}
\hline Probing signal & ID & $m s b$ & Signal & ID & $m s b$ & Signal & ID & $m s b$ & Signal & ID & $m s b$ \\
\hline$S_{I D}=1$ & $A 1$ & 0.5909 & $S_{1 D}=7$ & $B 3$ & 0.5906 & $S_{I D}=13$ & $D 1$ & 0.5905 & $S_{I D}=19$ & E3 & 0.5906 \\
\hline$S_{I D}=2$ & $A 2$ & 0.5908 & $S_{I D}=8$ & $B 4$ & 0.5905 & $S_{I D}=14$ & $D 2$ & 0.5905 & $S_{I D}=20$ & E4 & 0.5907 \\
\hline$S_{I D}=3$ & $A 3$ & 0.5908 & $S_{I D}=9$ & $\mathrm{C} 1$ & 0.5905 & $S_{I D}=15$ & D3 & 0.5905 & $S_{I D}=21$ & $F 1$ & 0.5907 \\
\hline$S_{I D}=4$ & A4 & 0.5907 & $S_{I D}=10$ & $C 2$ & 0.5905 & $S_{I D}=16$ & D4 & 0.5905 & $S_{I D}=22$ & $F 2$ & 0.5908 \\
\hline$S_{I D}=5$ & $B 1$ & 0.5907 & $S_{I D}=11$ & $\mathrm{C} 3$ & 0.5905 & $S_{I D}=17$ & $E 1$ & 0.5905 & $S_{I D}=23$ & F3 & 0.5908 \\
\hline$S_{1 D}=6$ & $B 2$ & 0.5906 & $S_{I D}=12$ & $\mathrm{C} 4$ & 0.5905 & $S_{I D}=18$ & $E 2$ & 0.5906 & $S_{I D}=24$ & F4 & 0.5909 \\
\hline
\end{tabular}




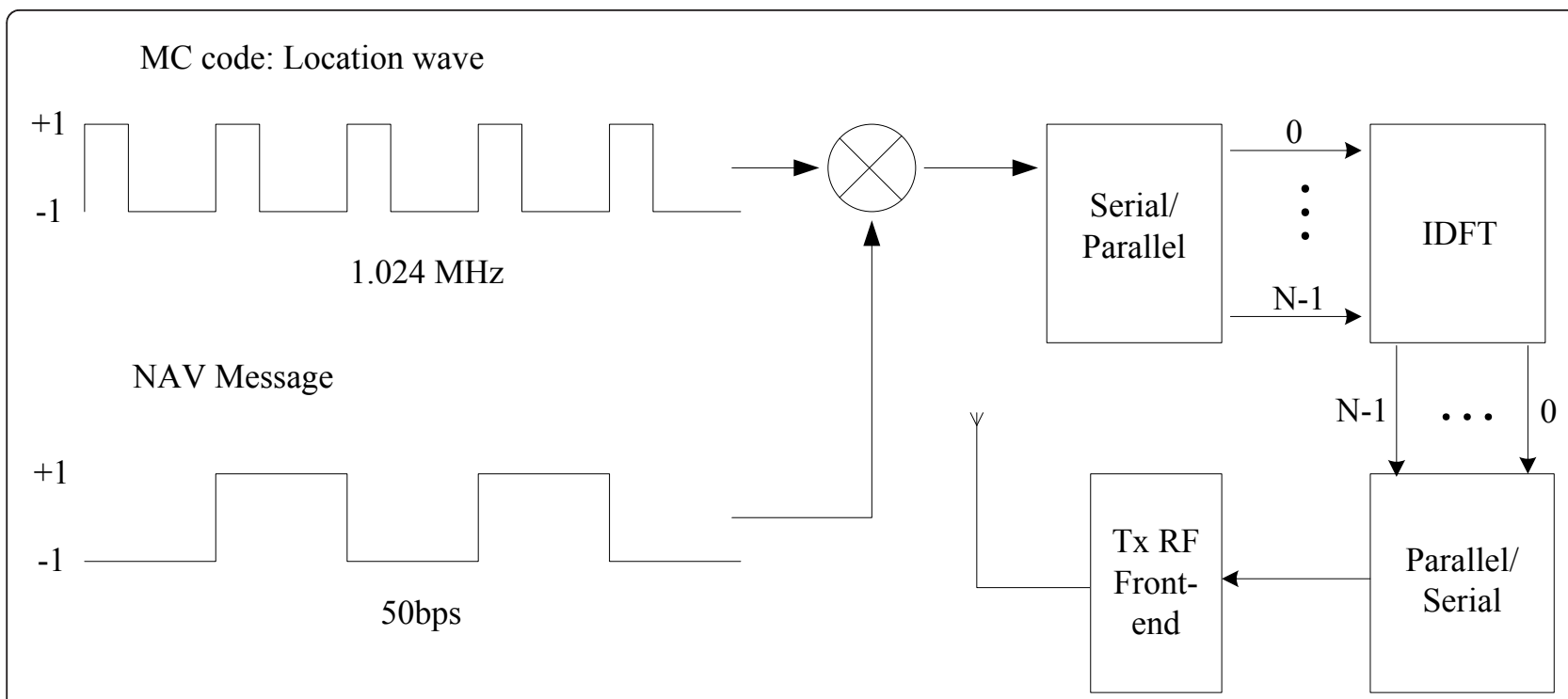

Figure 3 The transmission of the proposed MC code at certain SV, where the number of subcarrier slots $N=1024$ and the transmitted MC code is different for a different SV as given in (2) and Figure 2.

serial modulated data is converted to the parallel data and then transformed to the time domain by using inverse discrete Fourier transform (IDFT). Finally, the time-domain data is sent out by Tx RF Front-end. One note to be pointed out, every $20 \mathrm{MC}$-code cycles, i.e. $20 \mathrm{~ms}$, are modulated by the same one bit of the navigation message since the data rate of the MC code is much greater than that of the navigation message. The transmitted MC code is defined by Equations (1) and (2) and shown in Figure 2. The transmitted MC code is unique for a certain SV.

Figure 4 shows the block diagram of the reception of the proposed MC code in terms of range estimation. The time-domain data consisting of the modulated $\mathrm{MC}$ code is received by Rx RF Front-end. Furthermore, the serial modulated data is converted to the parallel data and then transformed to the frequency domain by using discrete Fourier transform (DFT).

The signal is received by Rx RF Front-end and downconverted to a serial baseband signal. The baseband signal is sampled and converted to a parallel data. Passing by DFT, the parallel data is tranformed to frequency domain and passed into parallel/serial converter. The output serial signal, together with the local code generator and local oscillator, is then used for Doppler removal and acquisition [12]. Furthermore, via signal tracking process [12], both the partial delay estimation $\hat{\tau}$ and NAV are obtained. Given $\hat{\tau}$ and NAV, the range between transmitter and receiver can be obtained. One note to be pointed out is that the cross-correlation in signal acquistion and tracking is implemented in the frequency domain, which is given in [20].

\section{$5 \mathrm{NBI}$ effect}

In this section, define the POC as the probability that the NBI hits any part of the mainlobe ${ }^{\mathrm{e}}$ of any of the subcarriers. The observation interval (OBI) is selected as $1 \mathrm{~ms}$ to evaluate NBI effect, leading to a null-to-null bandwidth of each subcarrier $w_{n n}=2 \mathrm{kHz}$. The frequency range out of the available bandwidth $2 B=2.048$ $\mathrm{MHz}$, where the probing signal might be hit by a NBI, is $w_{c}=42 w_{n n} \mathrm{kHz}$. This is the worst case since the greater the OBI, the narrower the subcarrier band, so the smaller the POC. The NBI effect is represented using POC, where $M$ uncorrelated NBIs are considered, $M=1,2, \ldots, 8$, and each NBI is referred to be a frequency tone [21].

Therefore, the resulting POC, $p_{c}$, can be obtained as

$$
p_{c}=1-\left(1-\frac{w_{c}}{2 B}\right)^{M}=1-0.959^{M} .
$$

Based on (8), let us evaluate the probability that there are at least four visible non-collision SVs for one GPS receiver since GPS positioning requires four SVs. Without loss of generality, assuming that there are eight SVs that are visible to one GPS receiver, the probability that there exists at most three visible non-colliding SVs is obtained as

$$
\begin{aligned}
p_{c c} & =\sum_{k=0}^{3} C_{8}^{k}\left(1-p_{c}\right)^{k} p_{c}^{8-k} \\
& =\sum_{k=0}^{3} C_{8}^{k} 0.959^{M k}\left[1-0.959^{M}\right]^{8-k},
\end{aligned}
$$




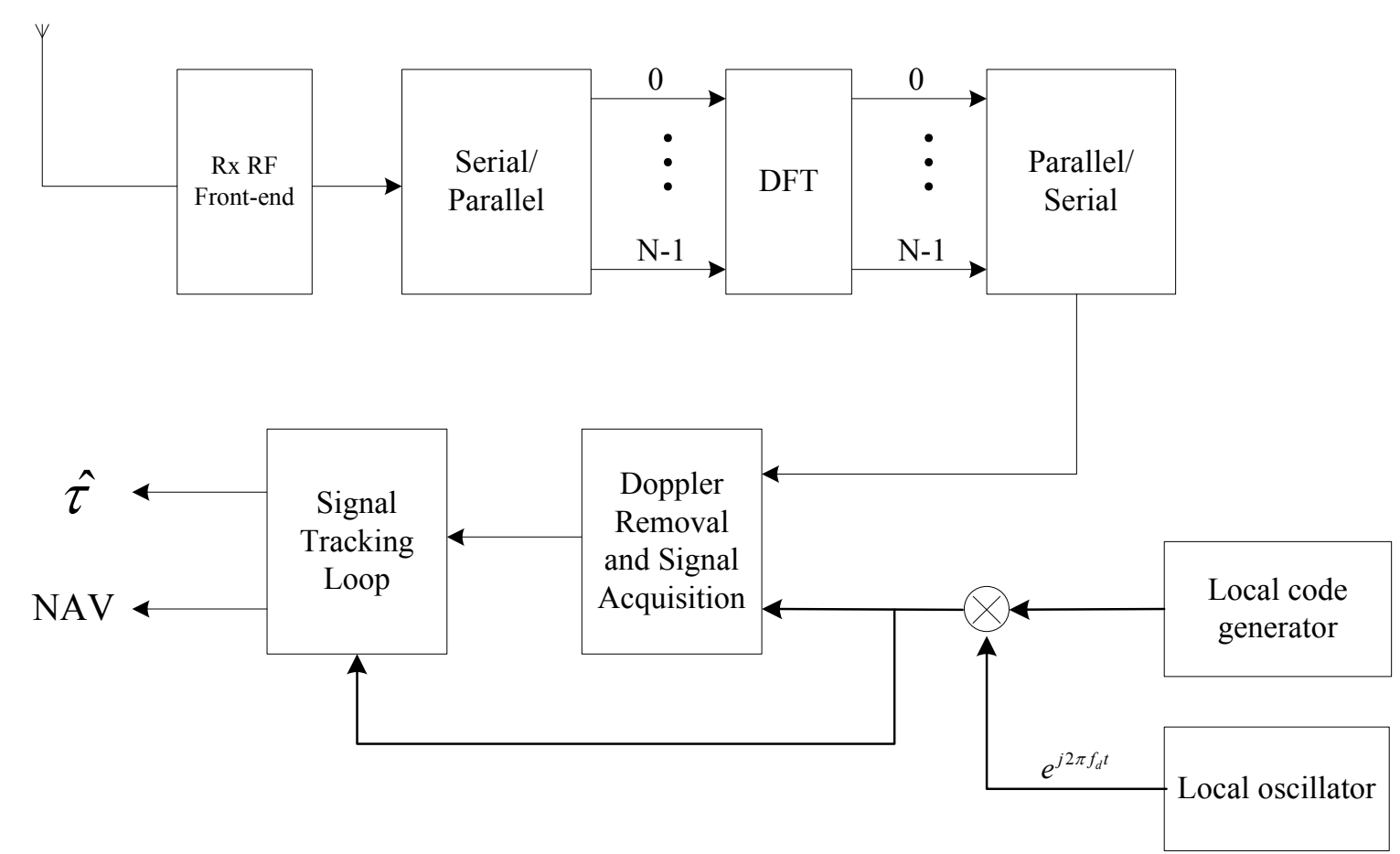

Figure 4 The reception of the proposed MC code at certain SV and its delay estimation $\hat{\tau}$, i.e. the range $\hat{R}=c \hat{\tau}, c$ is the speed of light, where the number of subcarrier slots $N=1024$ and the transmitted MC code is different for a different SV as given in (2) and Figure 2 .

where $C_{8}^{k}$ denotes the number of combinations for choosing $k$ out of eight SVs. And the probability that there exists at least four visible non-colliding SVs, i.e. the probability of positioning success, $P_{s}$, is obtained as

$$
P_{s}=1-p_{c c}=1-\sum_{k=0}^{3} C_{8}^{k} 0.959^{M k}\left[1-0.959^{M}\right]^{8-k}(10)
$$

For example, when $M=1$, we have $p_{c}=\frac{w_{c}}{2 B}=4.1 \%$ and $p_{c c}=\sum_{k=0}^{3} C_{8}^{k}\left(1-p_{c}\right)^{k} p_{c}^{8-k}=5.85 \times 10^{-6}$, so $P_{s} \approx$ $100 \%$, indicating that the proposed MC code is robust against NBI.

\section{Doppler effect}

The motion of each SV causes a Doppler shift in the transmitted $\mathrm{MC}$ codes which can force a collision between the various transmitted groups of MC codes that are received by a GPS receiver. For a thorough analysis on this collision, Appendix 1 analyzes the Doppler shift for an in-plane GPS receiver while Appendix 2 analyzes the Doppler shift for an off-plane GPS receiver in detail. In this section, the POC between MC codes due to Doppler shifts is theoretically explored. More specifically, we assume that a stationary GPS receiver is set to receive an MC code transmitted by SV "X", and measure the probability that its mainlobe collides with the mainlobe of MC codes transmitted by other SVs. In our analysis, we assume that the receiver has the maximum possible visible region, i.e. $\alpha=153^{\circ}$ as shown in Figure 5 in Appendix 2, in all of the six orbital planes, i. e. that the receiver experiences the maximum possible Doppler shift from each one of the six orbital planes at the same time. This is a worst case scenario, which can be used as an upper bound on POC.

\subsection{SV constellation}

In GPS, the constellation of SVs in each orbit is determined by true anomaly [12]. Since the eccentricity of orbits in GPS is less than 0.02 [12], which indicates a circle, the mean anomaly is approximately equal to the true anomaly. Therefore, by means of the mean anomaly [12], the constellation of SVs is shown in Figure 6.

\subsubsection{Collision analysis between SVs in one orbit}

Since the central angle between any two SVs in one orbit is fixed and the Doppler shift is monotonically decreasing with the central angle, then the Doppler difference (DD) between any two visible SVs has a small variational range. Furthermore, since the subcarriers allocated to each SV are predetermined, thus the frequency spacing between the subcarriers without Doppler shift (FSBD) is fixed. As long as the frequency spacing 


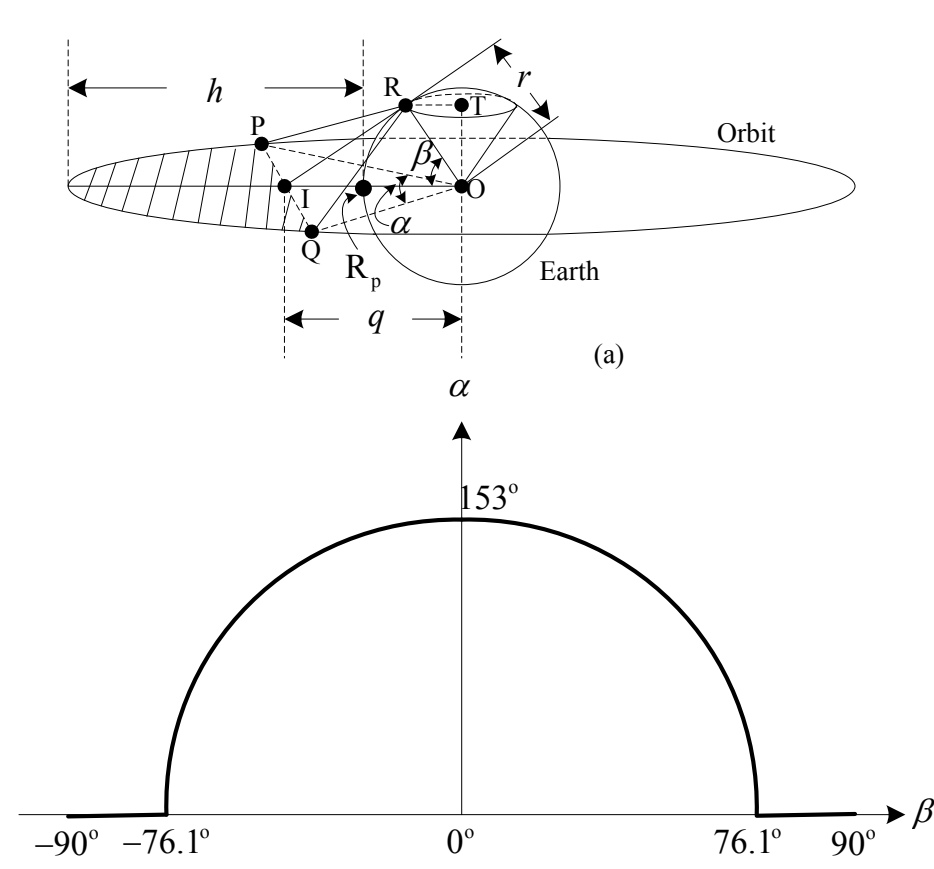

(b)

Figure 5 Visible range of an off-plane GPS receiver in terms of one SV (a) Representation of the visible range $\alpha$ of an off-plane GPS receiver; (b) the visible range $\alpha$ as a function of the elevation angle $\beta$.

after Doppler shift (FSAD) is larger than the frequency resolution, the collision between subcarriers shall be avoided, otherwise, a collision shall occur. Table 2 takes all pairs of SVs in one orbit into consideration with respect to their relative central angle, their DD, FSBD, FSAD and finally their decision outcome. In Table 2, the central angle is obtained from the constellation of SVs, DD is obtained from simulation, FSAD is obtained as the absolute value of summation of FSBD and DD, and the maximum frequency resolution (MFR) is selected as $1 \mathrm{kHz}$ corresponding to an OBI of $1 \mathrm{~ms}$. Even though, the central angles in Table 2 are less than the angle for the maximum possible visible range, one can conclude that there can never be any collision between groups of MC codes that are transmitted by SVs in one orbit.
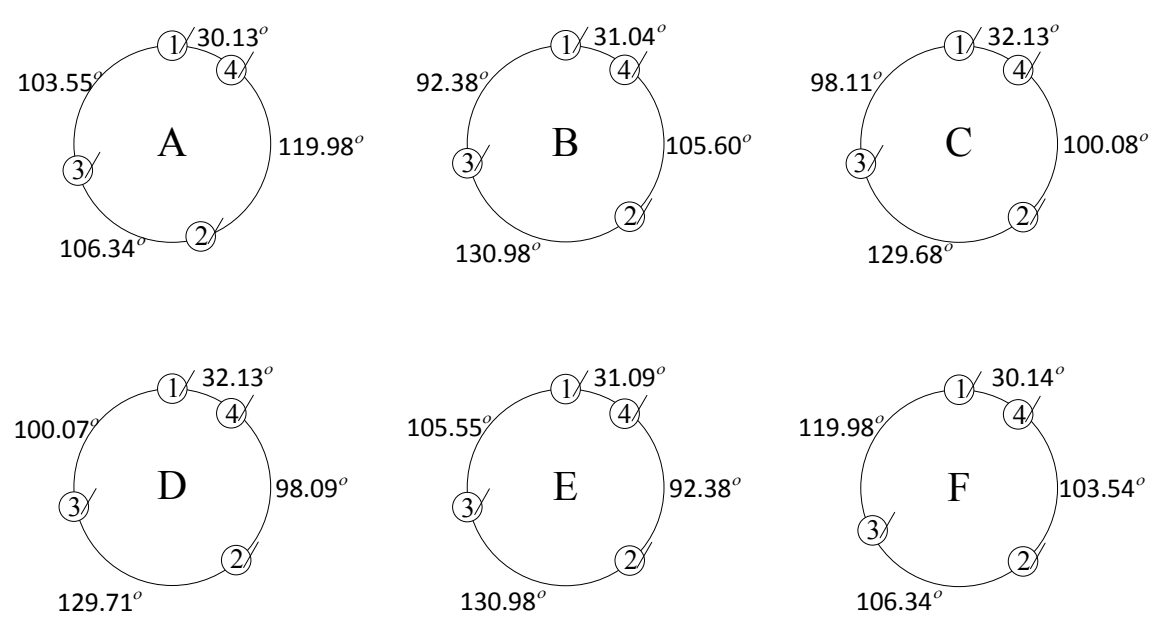

Figure 6 The constellation of SVs in each orbit based on the true anomaly 
Table 2 Collision analysis between SVs in each orbit for in-plane receivers, where Collision (S: Sure or N: Never) and CA: central angle

\begin{tabular}{|c|c|c|c|c|c|c|c|}
\hline Orbit & Slot ID & $C A$ & $\mathrm{DD}, \mathrm{kHz}$ & FSBD, $\mathrm{kHz}$ & FSAD, kHz & MFR, kHz & Collision \\
\hline \multirow[t]{6}{*}{ A } & $A 1-A 2$ & $150.11^{\circ}$ & -9.5 & 2 & 7.5 & 1 & $\mathrm{~N}$ \\
\hline & $A 1-A 3$ & $103.55^{\circ}$ & {$[7.5,8.5]$} & 4 & {$[11.5,12.5]$} & 1 & $\mathrm{~N}$ \\
\hline & $A 1-A 4$ & $30.13^{\circ}$ & {$[-3.2,-0.7]$} & 6 & {$[2.8,5.3]$} & 1 & N \\
\hline & $A 2-A 3$ & $106.34^{\circ}$ & {$[-8.6,-7.7]$} & 2 & {$[5.7,6.6]$} & 1 & N \\
\hline & $A 2-A 4$ & $119.98^{\circ}$ & {$[8.6,9.1]$} & 4 & {$[12.6,13.1]$} & 1 & $\mathrm{~N}$ \\
\hline & A3-A4 & $133.68^{\circ}$ & {$[-9.4,-9.2]$} & 2 & {$[7.2,7.4]$} & 1 & $\mathrm{~N}$ \\
\hline \multirow[t]{6}{*}{ B } & $B 1-B 2$ & $136.64^{\circ}$ & {$[-9.4,-9.3]$} & 2 & {$[7.3,7.4]$} & 1 & $\mathrm{~N}$ \\
\hline & $B 1-B 3$ & $92.38^{\circ}$ & {$[6.5,8.0]$} & 4 & {$[10.5,12.0]$} & 1 & N \\
\hline & $B 1-B 4$ & $31.04^{\circ}$ & {$[-3.3,-0.7]$} & 6 & {$[2.7,5.3]$} & 1 & $\mathrm{~N}$ \\
\hline & $B 2-B 3$ & $130.98^{\circ}$ & {$[-9.3,-9.1]$} & 2 & {$[7.1,7.3]$} & 1 & N \\
\hline & $B 2-B 4$ & $105.60^{\circ}$ & {$[7.6,8.6]$} & 4 & {$[11.6,12.6]$} & 1 & $\mathrm{~N}$ \\
\hline & $B 3-B 4$ & $123.42^{\circ}$ & {$[-9.2,-8.8]$} & 2 & {$[6.8,7.2]$} & 1 & N \\
\hline \multirow[t]{6}{*}{ C } & $C 1-C 2$ & $132.21^{\circ}$ & {$[-9.3,-9.2]$} & 2 & {$[7.2,7.3]$} & 1 & $N$ \\
\hline & $\mathrm{C} 1-\mathrm{CB}$ & $98.11^{\circ}$ & {$[7.0,8.3]$} & 4 & {$[11.0,12.3]$} & 1 & $\mathrm{~N}$ \\
\hline & $\mathrm{C} 1-\mathrm{C} 4$ & $32.13^{\circ}$ & {$[-3.4,-0.8]$} & 6 & {$[2.6,5.2]$} & 1 & $\mathrm{~N}$ \\
\hline & $C 2-C 3$ & $129.68^{\circ}$ & {$[-9.3,-9.1]$} & 2 & {$[7.1,7.3]$} & 1 & $\mathrm{~N}$ \\
\hline & $C 2-C 4$ & $100.08^{\circ}$ & {$[7.2,8.4]$} & 4 & {$[11.2,12.4]$} & 1 & N \\
\hline & C3-C4 & $130.24^{\circ}$ & {$[-9.3,-9.1]$} & 2 & {$[7.1,7.3]$} & 1 & $\mathrm{~N}$ \\
\hline \multirow[t]{6}{*}{ D } & $D 1-D 2$ & $130.22^{\circ}$ & {$[-9.3,-9.1]$} & 2 & {$[7.1,7.3]$} & 1 & $\mathrm{~N}$ \\
\hline & D1-D3 & $100.07^{\circ}$ & {$[7.2,8.4]$} & 4 & {$[11.2,12.4]$} & 1 & N \\
\hline & D1-D4 & $32.13^{\circ}$ & {$[-3.4,-0.8]$} & 6 & {$[2.6,5.2]$} & 1 & N \\
\hline & D2-D3 & $129.71^{\circ}$ & {$[-9.3,-9.1]$} & 2 & {$[7.1,7.3]$} & 1 & N \\
\hline & D2-D4 & $98.09^{\circ}$ & {$[7.0,8.3]$} & 4 & {$[11.0,12.3]$} & 1 & $\mathrm{~N}$ \\
\hline & D3-D4 & $132.20^{\circ}$ & {$[-9.3,-9.2]$} & 2 & {$[7.2,7.3]$} & 1 & $\mathrm{~N}$ \\
\hline \multirow[t]{6}{*}{ E } & $E 1-E 2$ & $123.47^{\circ}$ & {$[-9.2,-8.8]$} & 2 & {$[6.8,7.2]$} & 1 & N \\
\hline & $E 1-E 3$ & $105.55^{\circ}$ & {$[7.6,8.6]$} & 4 & {$[11.6,12.6]$} & 1 & N \\
\hline & $E 1-E 4$ & $31.09^{\circ}$ & {$[-3.3,-0.7]$} & 6 & {$[2.7,5.3]$} & 1 & N \\
\hline & $E 2-E 3$ & $130.98^{\circ}$ & {$[-9.3,-9.1]$} & 2 & {$[7.1,7.3]$} & 1 & $\mathrm{~N}$ \\
\hline & $E 2-E 4$ & $92.38^{\circ}$ & {$[6.5,8.0]$} & 4 & {$[10.5,12.0]$} & 1 & N \\
\hline & $E 3-E 4$ & $136.64^{\circ}$ & {$[-9.4,-9.3]$} & 2 & {$[7.3,7.4]$} & 1 & N \\
\hline \multirow[t]{6}{*}{$\mathrm{F}$} & $F 1-F 2$ & $133.68^{\circ}$ & {$[-9.4,-9.2]$} & 2 & {$[7.2,7.4]$} & 1 & N \\
\hline & $F 1-F 3$ & $119.98^{\circ}$ & {$[8.6,9.1]$} & 4 & {$[12.6,13.1]$} & 1 & $\mathrm{~N}$ \\
\hline & $F 1-F 4$ & $30.14^{\circ}$ & {$[-3.2,-0.7]$} & 6 & {$[2.8,5.3]$} & 1 & $\mathrm{~N}$ \\
\hline & $F 2-F 3$ & $106.34^{\circ}$ & {$[-8.6,-7.7]$} & 2 & {$[5.7,6.6]$} & 1 & N \\
\hline & $F 2-F 4$ & $103.54^{\circ}$ & {$[7.5,8.5]$} & 4 & {$[11.5,12.5]$} & 1 & $\mathrm{~N}$ \\
\hline & F3-F 4 & $150.12^{\circ}$ & -9.5 & 2 & 7.5 & 1 & N \\
\hline
\end{tabular}

\subsubsection{Analysis On the visibility of an SV}

It is essential to analyze the visibility of an SV by the receiver in order to obtain its probability of visibility and hence its POC with other SVs. Since the maximum possible visible range for one $\mathrm{SV}$ by the receiver is $153^{\circ}$, according to the constellation shown in Figure 6, the visible region denoted by the central angle can be obtained. Take $B 2$ as an example, moving clockwise for a given receiver. When $B 3$ descends past the horizon, $B 2$ is the only visible $\mathrm{SV}$ in orbit $B$ until $B 4$ ascends past the horizon, which corresponds to $130.98^{\circ}+105.60^{\circ}-153^{\circ}=83.58^{\circ}$. Furthermore, take the combination of $C 1$ and $C 3$ as an example, both moving clockwise for a given receiver. When $C 4$ descends past the horizon, only $C 1$ and $C 3$ in orbit $C$ are visible to the receiver until $C 1$ descends past the horizon, which corresponds to $32.13^{\circ}$. Table 3 illustrates all possible combinations of SVs as well as their corresponding visible regions and percentages. In Table 3 , the probability of visibility (POV) is given as the ratio of the visible region to the whole circle since each SV is assumed to move with a constant velocity.

\subsubsection{Approximation of visibility}

From Table 3 that, one can see that the scenarios for all six orbits are similar. Firstly, the combinations of invisible SVs are exactly the same for all 6 orbits. Secondly, for all six orbits, the 2 nd SV, the 3rd SV and the combination of 
Table 3 Visibility analysis for the fixed in-plane GPS receiver, where VI: Visible interval, POV: Probability of Visibility, APOV: Average Probability of Visibility and OX: Orbit $X$, where $X$ denotes $A, B, C, D, E$ or $F$

\begin{tabular}{|c|c|c|c|c|c|c|c|c|}
\hline$\overline{O A}$ & $A 2$ & A3 & $A 1, A 3$ & $A 1, A 4$ & $A 2, A 3$ & $A 2, A 4$ & $A 1, A 2, A 4$ & $A 1, A 3, A 4$ \\
\hline$\overline{\mathrm{Vl}}$ & $73.32^{\circ}$ & $56.89^{\circ}$ & $30.13^{\circ}$ & $100.06^{\circ}$ & $46.66^{\circ}$ & $30.13^{\circ}$ & $2.89^{\circ}$ & $19.32^{\circ}$ \\
\hline POV & $20.40 \%$ & $15.80 \%$ & $8.40 \%$ & $28 \%$ & $13 \%$ & $8.40 \%$ & $0.80 \%$ & $5.40 \%$ \\
\hline$O B$ & $B 2$ & B3 & $B 1, B 3$ & $B 1, B 4$ & $B 2, B 3$ & $B 2, B 4$ & $B 1, B 2, B 4$ & $B 1, B 3, B 4$ \\
\hline $\mathrm{Vl}$ & $83.58^{\circ}$ & $70.36^{\circ}$ & $31.04^{\circ}$ & $76.02^{\circ}$ & $22.02^{\circ}$ & $31.04^{\circ}$ & $16.36^{\circ}$ & $29.58^{\circ}$ \\
\hline POV & $23.20 \%$ & $19.50 \%$ & $8.60 \%$ & $21.20 \%$ & $6.10 \%$ & $8.60 \%$ & $4.50 \%$ & $8.20 \%$ \\
\hline$O C$ & $C 2$ & C3 & $C 1, C 3$ & $C 1, C 4$ & $C 2, C 3$ & $C 2, C 4$ & $C_{1}, C_{2}, C_{4}$ & $\mathrm{C} 1, \mathrm{C} 3, \mathrm{C} 4$ \\
\hline $\mathrm{Vl}$ & $76.76^{\circ}$ & $74.79^{\circ}$ & $32.13^{\circ}$ & $77.32^{\circ}$ & $23.32^{\circ}$ & $32.13^{\circ}$ & $20.79^{\circ}$ & $22.76^{\circ}$ \\
\hline POV & $21.30 \%$ & $20.80 \%$ & $8.90 \%$ & $21.50 \%$ & $6.50 \%$ & $8.90 \%$ & $5.80 \%$ & $6.30 \%$ \\
\hline$O D$ & D2 & D3 & $D 1, D 3$ & $D 1, D 4$ & $D 2, D 3$ & $D 2, D 4$ & $D 1, D 2, D 4$ & $D 1, D 3, D 4$ \\
\hline $\mathrm{Vl}$ & $74.80^{\circ}$ & $76.78^{\circ}$ & $32.13^{\circ}$ & $77.29^{\circ}$ & $23.29^{\circ}$ & $32.13^{\circ}$ & $22.78^{\circ}$ & $20.80^{\circ}$ \\
\hline POV & $20.80 \%$ & $21.30 \%$ & $8.90 \%$ & $21.50 \%$ & $6.50 \%$ & $8.90 \%$ & $6.30 \%$ & $5.80 \%$ \\
\hline$O E$ & $E 2$ & E3 & $E 1, E 3$ & $E 1, E 4$ & $E 2, E 3$ & $E 2, E 4$ & $E 1, E 2, E 4$ & $E 1, E 3, E 4$ \\
\hline $\mathrm{Vl}$ & $70.36^{\circ}$ & $83.53^{\circ}$ & $31.09^{\circ}$ & $76.02^{\circ}$ & $22.02^{\circ}$ & $31.09^{\circ}$ & $29.53^{\circ}$ & $16.36^{\circ}$ \\
\hline POV & $19.50 \%$ & $23.20 \%$ & $8.60 \%$ & $21.20 \%$ & $6.10 \%$ & $8.60 \%$ & $8.20 \%$ & $4.50 \%$ \\
\hline $\mathrm{OF}$ & $F 2$ & F3 & $F 1, F 3$ & $F 1, F 4$ & $F 2, F 3$ & $F 2, F 4$ & $F 1, F 2, F 4$ & $F 1, F 3, F 4$ \\
\hline $\mathrm{Vl}$ & $56.88^{\circ}$ & $73.32^{\circ}$ & $30.14^{\circ}$ & $100.06^{\circ}$ & $46.66^{\circ}$ & $30.14^{\circ}$ & $19.32^{\circ}$ & $2.88^{\circ}$ \\
\hline POV & $15.80 \%$ & $20.40 \%$ & $8.40 \%$ & $28 \%$ & $13 \%$ & $8.40 \%$ & $5.40 \%$ & $0.80 \%$ \\
\hline APOV & $20.10 \%$ & $20.10 \%$ & $8.50 \%$ & $23.50 \%$ & $8.40 \%$ & $8.50 \%$ & $5.10 \%$ & $5.10 \%$ \\
\hline
\end{tabular}

the 1st and 4th SV have similarly the largest visible interval, which corresponds to a POV of approximately $20 \%$. Thirdly, for all orbits, the majority of combinations of the 1st and 3rd SV, the 2nd and 3rd SV, the 2nd and 4th SV, the 1st, 2nd and 4th SV, and the 1st, 3rd and 4th SV have a small POV that is lower than $10 \%$. In other words, even though they are different in some ways, e.g. the combinations of $A 2$ and $A 3$, as well as $F 2$ and $F 3$ have about $13 \%$ $\mathrm{POV}$, the scenarios for all six orbits are still very similar. Therefore, to simplify the analysis of the POC, the average visible interval and APOV in Table 3, are used for all six orbits. This is equivalent to using the average constellation for one orbit, which is shown in Figure 7, instead of the six constellations which are shown in Figure 6.

\subsection{Theoretical procedure for calculating POC}

Since there is no collision between SVs in one orbit, the in-orbit 'adjacencies ${ }^{\text {f }}$ do not exist and the number of 'adjacencies' is thus reduced. To calculate the POC: denote by $M$ the number of 'adjacencies' of the desired SV " $\mathrm{X}$ "; denote by, $p_{M_{k}}, k=1,2,3,4$, the probability of having $k$ 'adjacencies' to " $\mathrm{X}$ ", which is an a priori probability; denote by, $p_{v_{k j}}, 1 \leq j \leq k$, the probability of visibility of $j$ 'adjacencies' to "X" when $M=k$, which is also an a priori probability; denote by, $p_{k}$ (collision $\mid j$ visible), the POC under the condition that there are $j$ visible 'adjacencies' to " $\mathrm{X}$ " when $M=k$, which is a conditional probability.

When $M=1$ with a probability $p_{M_{1}}$, the procedure for calculating $p_{c_{1}}$, which is defined as the POC when there is only one 'adjacency', is as follows: (1) calculate the probability of visibility $p_{v_{11}}$ of this 'adjacency'; (2) calculate the conditional probability $p_{1}$ (collision $\mid 1$ visible), which is the POC when the 'adjacency' of SV " $\mathrm{X}$ " is also visible to the GPS receiver; (3) calculate $p_{c_{1}}$ by removing the condition with respect to the visibility:

$$
\left.p_{c_{1}}=p_{1} \text { (collision } \mid 1 \text { visible }\right) p_{v_{11}} \text {. }
$$

Similarly, When $M=2$ with a probability $p_{M_{2}}, p_{c_{2}}$, which is defined as the POC when there are two 'adjacencies', can be obtained as

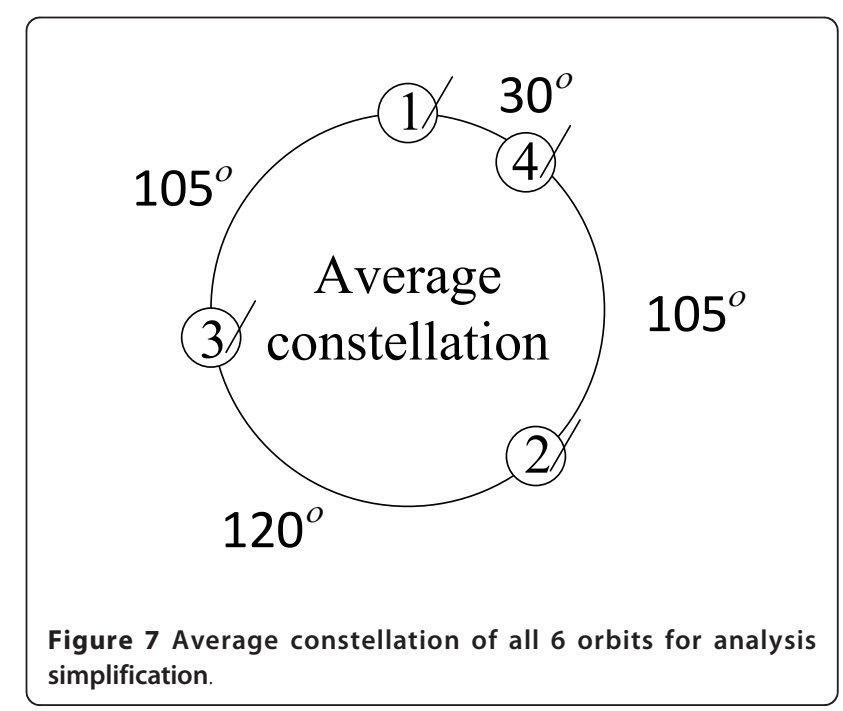




$$
\begin{aligned}
p_{c_{2}} & =p_{2}(\text { collision } \mid 1 \text { visible }) p_{v_{21}} \\
& +p_{2}(\text { collision } \mid 2 \text { visible }) p_{v_{22}} ;
\end{aligned}
$$

when $M=3$ with a probability $p_{M_{3},} p_{c_{3}}$, which is defined as the POC when there are three 'adjacencies', can be obtained as

$$
\begin{aligned}
p_{c_{3}} & =p_{3}(\text { collision } \mid 1 \text { visible }) p_{v_{31}} \\
& +p_{3}(\text { collision } \mid 2 \text { visible }) p_{v_{32}} \\
& +p_{3}(\text { collision } \mid 3 \text { visible }) p_{v_{33}} ;
\end{aligned}
$$

When $M=4$ with a probability $p_{M_{4}}$, the procedure for calculating $p_{c_{4}}$, which is defined as the POC when there are four 'adjacencies' can be obtained as

$$
\begin{aligned}
p_{c_{4}} & =p_{4}(\text { collision } \mid 1 \text { visible }) p_{v_{41}} \\
& +p_{4}(\text { collision } \mid 2 \text { visible }) p_{v_{42}} \\
& +p_{4}(\text { collision } \mid 3 \text { visible }) p_{v_{43}} \\
& +p_{4}(\text { collision } \mid 4 \text { visible }) p_{v_{44}} .
\end{aligned}
$$

The overall POC, $p_{c}$, is represented by removing the condition with respect to $M$ :

$$
p_{c}=\sum_{k=1}^{4} p_{c_{k}} p_{M_{k}} .
$$

\subsection{A priori probability $p_{v_{k j}}$}

Each SV has a unique allocation of subcarriers, and each subcarrier has different neighboring sub-carriers, some of which are co-orbital while others are not. We already know that, no collision occurs between co-orbital subcarriers. Therefore, the four SVs in one orbit have a different number of 'adjacencies' and correspondingly have different probabilities of collision at any given time. However, all first SVs in each one of the 6 orbits have the same scenario and the same number of 'adjacencies', which we cluster into Category I; all second SVs have the same scenario and the same number of 'adjacencies', which we cluster into Category II; all third SVs have the same scenario and the same number of 'adjacencies', which we cluster into Category III; all fourth SVs have the same scenario and the same number of 'adjacencies', which we cluster into Category IV. All of the SVs are therefore clustered into one of the four categories and are shown in Table 4.

The desired SV would locate in one of these four Categories. The following parts will consider all cases that the desired SV lies in a specific Catogory. In order to obtain the priori probability $p_{v_{k j}}$,

- the first step is to get the number of the 'adjacency' of the desired SV. Since the number of 'adjacencies' is evidently changing with the Doppler shift of the desired
Table 424 SVs are clustered into four categories, each of which contains six SVs

\begin{tabular}{ll}
\hline Categories & sVs \\
\hline I & $A 1, B 1, C 1, D 1, E 1, F 1$ \\
$\|$ & $A 2, B 2, C 2, D 2, E 2, F 2$ \\
III & $A 3, B 3, C 3, D 3, E 3, F 3$ \\
IV & $A 4, B 4, C 4, D 4, E 4, F 4$ \\
\hline
\end{tabular}

SV, the number of 'adjacencies' will be obtained with the corresponding Doppler interval of the desired SV.

- For the case that the desired SV has one 'adjacency', referring to Table 3, the visiable range of this 'adjacency' in the percentage can be obtained. This value is $p_{v_{11}}$.

- For the case that the desired SV has two 'adjacencies', referring to Table 3, the visible range of either of two 'adjacencies' can be obtained and this value leads to $p_{v_{21}}$. And the visible range of both 'adjacencies' obtained by reffereing to Table 3 will lead to $p_{v_{22}}$.

- For the case that the desired SV has three 'adjacencies', referring to Table 3, the visible range of any of three 'adjacencies' can be obtained and this value leads to $p_{v_{31}}$. The visible range of any two 'adjacencies' obtained by reffereing to Table 3 will lead to $p_{v_{32}}$. And the visible range of all three 'adjacencies' obtained by reffereing to Table 3 will lead to $p_{v_{33}}$.

- For the case that the desired SV has four 'adjacencies', referring to Table 3, the visible range of any of four 'adjacencies' can be obtained and this value leads to $p_{v_{41}}$. The visible range of any two 'adjacencies' obtained by reffereing to Table 3 will lead to $p_{v_{42}}$. The visible range of any three 'adjacencies' obtained by reffereing to Table 3 will lead to $p_{v_{43}}$. And the visible range of all four 'adjacencies' obtained by reffereing to Table 3 will lead to $p_{v_{44}}$.

The details are presented as follows.

\subsubsection{Category I}

Let the desired SV be in Category I and be referred to as $B 1$. When $B 1$ suffers from a Doppler shift, $f_{D_{X}}$, which falls in the interval of $[-4.75,-3.25] \mathrm{kHz}$, we have four 'adjacencies' to $B 1$ which might collide with $B 1$ : SVs $A 1$, $A 2, A 3$ and $A 4$. More specifically, because the subcarrier spacing between $B 1$ and $A 4$ is $2 \mathrm{kHz}, A 4$ will completely collide with $B 1$ if the Doppler shift on $A 4$ is $f_{D_{X}}+2 \mathrm{kHz}$. Similarly, $A 3$ will completely collide with $B 1$ if the Doppler shift on $A 3$ is $f_{D_{X}}+4 \mathrm{kHz} . A 2$ will also completely collide with $B 1$ if the Doppler shift on A2 is $f_{D_{X}}+6 \mathrm{kHz}$. Finally, A1 will also completely collide with $B 1$ if the Doppler shift on $A 1$ is $f_{D_{X}}+8 \mathrm{kHz}$. When the Doppler shift on $B 1$ falls in the interval of [-3.25, - 1.25$] \mathrm{kHz}, A 1, A 2$ and $A 3$ are its 'adjacencies', however, $A 1$ is not capable of colliding with $B 1$ even 
with the biggest possible Doppler shift. When $B 1$ suffers from a Doppler shift which falls in the interval of $[-1.25$, $0.75] \mathrm{kHz}, A 3$ and $A 4$ are its 'adjacencies'. When $B 1$ suffers from a Doppler shift which falls in the interval of $[0.75,2.75] \mathrm{kHz}$, only $A 4$ is its 'adjacency'. When the Doppler shift on $B 1$ is in the interval of [2.75, 3.25] $\mathrm{kHz}$, no 'adjacencies' exist and so no collision can take place. Finally, when the Doppler shift on $B 1$ belongs to the interval [3.25, 4.75] kHz, C1 becomes its 'adjacency' and can collide with it. Table 5 shows the number of 'adjacencies' of the desired SV in Category I with respect to $f_{D_{X}}$.

Referring to the average visible interval, the a priori probabilities can be obtained. Firstly, if $f_{D_{X}} \in[3.25,4.75] \mathrm{kHz}$, only $C 1$ is the 'adjacency' of $B 1$. Looking into Table $3, C 1$ is visible in the form of the following four combinations: $\{C 1, C 3\},\{C 1, C 4\},\{C 1$, $C 2, C 4\}$, or $\{C 1, C 3, C 4\}$. In total, the visible range for $C 1$ is $8.5 \%+23.5 \%+5.1 \% \times 2=42.2 \%$. Similarly, when $f_{D_{X}} \in[0.75,2.75] \mathrm{kHz}$, the visible range is also $42.2 \%$. Overall, $p_{v_{11}}=42.2 \%$.

When $f_{D_{X}} \in[-1.25,0.75] \mathrm{kHz}$, there are two 'adjacencies' for $B 1: A 3$ and $A 4$. Looking into Table 3, exactly one 'adjacency', $A 3$, is visible in the following three forms: $A 3$ alone, the combinations of $\{A 1, A 3\}$ or $\{A 2, A 3\}$. In this case, $A 3$ has a visible range of $37 \%$. Similarly, exactly one 'adjacency', $A 4$, is visible in the following three forms: the combinations of $\{A 1, A 4\}$, $\{A 2, A 4\}$, or $\{A 1, A 2, A 4\}$. In this case, $A 4$ has a visibility range of $37.1 \%$. Finally, exactly two 'adjacencies' are visible in the form of the combination of $\{A 1, A 3, A 4\}$, which corresponds to a visibility range of $5.1 \%$. Thus, it is concluded that $p_{v_{21}}=74.1 \%$ and $p_{v_{22}}=5.1 \%$.

When $f_{D_{X}} \in[-3.25,-1.75] \mathrm{kHz}$, there are three 'adjacencies' $A 2, A 3$ and $A 4$ of the desired SV $B 1$. Looking into Table 3 , exactly one 'adjacency' $A 2$ is visible in the form of $A 2$ alone, which corresponds to a visibility range of $20.1 \%$. Similarly, exactly one 'adjacency' $A 3$ is visible in the form of $A 3$ alone or as a combination of $\{A 1, A 3\}$, which corresponds to a visibility range of $28.6 \%$. Also, exactly one 'adjacency' $A 4$ is visible in the form of the combination of $\{A 1, A 4\}$, which corresponds to a visibility range of $23.5 \%$. Exactly two 'adjacencies' $A 2$ and $A 3$ are visible with a visibility range of $5.1 \%$. Exactly two 'adjacencies' $A 2$ and $A 4$ are visible in the form of the two combinations of $\{A 2, A 4\}$ or $\{A 1, A 2$, $A 4\}$, which correspond to a visibility range of $13.6 \%$. Exactly two 'adjacencies' $A 3$ and $A 4$ are visible in the form of the combination of $\{A 1, A 3, A 4\}$, which corresponds to a visibility range of $5.1 \%$. Exactly three 'adjacencies' $A 2, A 3$ and $A 4$ are always invisible. Thus, it is concluded that $p_{v_{31}}=72.2 \%, \quad p_{v_{32}}=23.8 \%$ and $p_{v_{33}}=0 \%$.

When $f_{D_{X}} \in[-4.75,-3.25] \mathrm{kHz}$, there are four 'adjacencies' for $B 1$ : $A 1, A 2, A 3$ and $A 4$. Looking into Table 3 , exactly one 'adjacency' is visible in the form of $A 2$ or $A 3$, which corresponds to the visibility range of $40.2 \%$. Exactly two 'adjacencies' are visible in the form of $\{A 1$, $A 3\},\{A 1, A 4\},\{A 2, A 3\}$ or $\{A 2, A 4\}$, which correspond to a visibility range of $48.9 \%$. Exactly three 'adjacencies' are visible in the form of $\{A 1, A 2, A 4\}$ or $\{A 1, A 3, A 4\}$, which correspond to a visibility range of $10.2 \%$. One should note that it is impossible that exactly four 'adjacencies' are visible simultaneously. Thus, it is concluded that $p_{v_{41}}=40.2 \%, \quad p_{v_{42}}=48.9 \%, \quad p_{v_{43}}=10.2 \%$ and $p_{v_{44}}=0 \%$.

\subsubsection{Category II}

Similarly, taking $B 2$ as an example of the desired SV, the number of 'adjacencies' of $B 2$ with respect to $f_{D_{X}}$ is shown in Table 6.

When $f_{D_{X}} \in[1.25,3.25] \mathrm{kHz}$, only $C 1$ is the 'adjacency' for B2. Similar to Category I, we have $p_{v_{11}}=42.2 \%$. When $f_{D_{X}} \in[-1.25,0.75] \mathrm{kHz}$, there is also only one 'adjacency' to $B 2: A 4$. Following a similar analysis, it is concluded that is also equal to

Table 5 The changing process of the number of 'adjacencies' of the desired SV "X" in Category I (i.e. the SV B1) with respect to Doppler shift on the SV " $\mathrm{X}$ "

\begin{tabular}{lllllll}
\hline$f_{D_{X}}, k H z$ & {$[-4.75,-3.25]$} & {$[-3.25,-1.25]$} & {$[-1.25,0.75]$} & {$[0.75,2.75]$} & {$[2.75,3.25]$} & {$[3.25,4.75]$} \\
$M$ & 4 & 3 & 2 & 1 & 0 & 1 \\
'adjacencies' & $A 1, A 2, A 3, A 4$ & $A 2, A 3, A 4$ & $A 3, A 4$ & $A 4$ & NULL & $C 1$ \\
\hline
\end{tabular}

Table 6 The changing process of the number of 'adjacencies' of the desired SV "X" in Category II (i.e. the SV B2) with respect to Doppler shift on the SV " $\mathrm{X}$ "

\begin{tabular}{llllll}
\hline$f_{D_{X},}, k H z$ & {$[-4.75,-3.25]$} & {$[-3.25,-1.25]$} & {$[-1.25,0.75]$} & {$[0.75,1.25]$} & {$[1.25,3.25]$} \\
$M$ & 3 & 2 & 1 & 0 & 1 \\
'adjacencies' & $A 2, A 3, A 4$ & $A 3, A 4$ & $A 4$ & NULL & $C 1$ \\
\hline
\end{tabular}


$f_{D_{X}}$. Consequently, the overall a priori probability for $M$ $=1$ is also $42.2 \%$.

When $f_{D_{X}} \in[3.25,4.75] \mathrm{kHz}, C 1$ and $C 2$ are its 'adjacencies'. Referring to Table 3, exactly one 'adjacency' $C 1$ is visible in the form of the combinations: $\{C 1, C 3\},\{C 1, C 4\}$, or $\{C 1, C 3, C 4\}$, which corresponds to a visibility range of $37.1 \%$. Exactly one 'adjacency' $C 2$ is visible in the form of $C 2$ alone, or in the combinations of $\{C 2, C 3\}$ or $\{C 2, C 4\}$, which corresponds to a visibility range of $37 \%$. Exactly two 'adjacencies' are simultaneously visible in the form of the combination of $\{C 1, C 2, C 4\}$, which corresponds to a visibility range of $5.1 \%$. Thus, it is concluded that $p_{v_{21}}=74.1 \%$ and $p_{v_{22}}=5.1 \%$. When $f_{D_{X}} \in[-3.25,-1.25] \mathrm{kHz}, A 3$ and $A 4$ are the 'adjacencies' of $B 2$. As analyzed in Category I, the same values of $p_{v_{21}}=74.1 \%$ and $p_{v_{22}}=5.1 \%$ are obtained. Overall speaking, when $M=2$, the a priori probabilities are $p_{v_{21}}=74.1 \%$ and $p_{v_{22}}=5.1 \%$.

When $f_{D_{X}} \in[-4.75,-3.25] \mathrm{kHz}$, there are three 'adjacencies' $A 2, A 3$ and $A 4$ of the desired SV $B 2$. With the same analysis shown in Category I, one can conclude that $p_{v_{31}}=72.2 \%, p_{v_{32}}=23.8 \%$ and $p_{v_{33}}=0 \%$. Because $B 2$ has at most three 'adjacencies', it is evident that $p_{v_{41}}=p_{v_{42}}=p_{v_{43}}=p_{v_{44}}=0 \%$.

\subsubsection{Category III}

Similarly, taking $B 3$ as an example of the desired SV, the number of 'adjacencies' of $B 3$ with respect to $f_{D_{X}}$ is shown in Table 7.

When $f_{D_{X}} \in[-0.75,1.25] \cup[-3.25,-1.25] \mathrm{kHz}$, only $C 1$ or $A 4$ is the 'adjacency' of the desired SV B3. As shown in Category II, it is obtained that $p_{v_{11}}=42.2 \%$.

When $f_{D_{X}} \in[1.25,3.25] \mathrm{kHz}, C 1$ and $C 2$ are the 'adjacencies' of the desired SV B3. As analyzed in Category II, it is provided that $p_{v_{21}}=74.1 \%$ and $p_{v_{22}}=5.1 \%$. When $f_{D_{X}} \in[-4.75,-3.25] \mathrm{kHz}$, there are also two 'adjacencies': $A 3$ and $A 4$, and it is shown that also $p_{v_{21}}=74.1 \%$ and $p_{v_{22}}=5.1 \%$. Overall speaking, when $M=2$, the a priori probabilities are $p_{v_{21}}=74.1 \%$ and $p_{v_{22}}=5.1 \%$.

When $f_{D_{X}} \in[3.25,4.75] \mathrm{kHz}, C 1, C 2$ and $C 3$ are the 'adjacencies' of the desired SV B3. Referring to Table 3, exactly one 'adjacency' $C 1$ is visible in the form of the combination $\{C 1, C 4\}$, which corresponds to a visibility range of $23.5 \%$. Exactly one 'adjacency' $C 2$ is visible in the form of $C 2$ alone or the combination $\{C 2, C 4\}$, which corresponds to a visibility range of $28.6 \%$. Exactly one 'adjacency' C3 is visible in the form of C3 alone, which corresponds to the visibility range of $20.1 \%$. Exactly two 'adjacencies' $C 1$ and $C 2$ are visible in the form of the combinations $\{C 1, C 2, C 4\}$, which corresponds to a visibility range of $5.1 \%$. Exactly two 'adjacencies' $\{C 2, C 3\}$ are visible with a visibility range of 8.4\%. Exactly two 'adjacencies' $C 1$ and $C 3$ are visible in the form of the combinations of $\{C 1, C 3\}$ or $\{C 1, C 3$, $A 4\}$, which correspond to a visibility range of $13.6 \%$. Exactly three 'adjacencies' $C 1, C 2$ and $C 3$ are always invisible. Thus, it is concluded that $p_{v_{31}}=72.2 \%$, $p_{v_{32}}=23.8 \%$ and $p_{v_{33}}=0 \%$. Also since $B 3$ has at most three 'adjacencies', it is evident that $p_{v_{41}}=p_{v_{42}}=p_{v_{43}}=p_{v_{44}}=0 \%$.

\subsubsection{Category IV}

Similarly, taking $B 4$ as an example of the desired SV, the number of 'adjacencies' of $B 4$ with respect to $f_{D_{X}}$ is shown in Table 8.

When $f_{D_{X}} \in[-4.75,-3.25] \cup[-2.75,-0.75] \mathrm{kHz}$, only $C 1$ or $A 4$ are the 'adjacencies' of $B 4$. As shown in Category III, it is obtained that $p_{v_{11}}=42.2 \%$. When $f_{D_{X}} \in[-0.75,1.25] \mathrm{kHz}, C 1$ and $C 2$ are the 'adjacencies' of B4. As shown in Category III, $p_{v_{21}}=74.1 \%$ and $p_{v_{22}}=5.1 \%$. When $f_{D_{X}} \in[1.25,3.25] \mathrm{kHz}$, the SVs $C 1$, $C 2$ and $C 3$ are the 'adjacencies' of $B 4$. As shown in Category III, $p_{v_{31}}=72.2 \%, p_{v_{32}}=23.8 \%$ and $p_{v_{33}}=0 \%$. When $f_{D_{X}} \in[3.25,4.75] \mathrm{kHz}$, there are four 'adjacencies' $C 1, C 2, C 3$ and $C 4$ of the desired SV $B 4$, which is the same as the four 'adjacencies' $A 1, A 2, A 3$ and $A 4$.

Table 7 The changing process of the number of 'adjacencies' of the desired SV " $X$ " in Category III (i.e. the SV B3) with respect to Doppler shift on the SV " $\mathrm{X}$ "

\begin{tabular}{lllllll}
\hline$f_{D_{X}}, k H z$ & {$[-4.75,-3.25]$} & {$[-3.25,-1.25]$} & {$[-1.25,-0.75]$} & {$[-0.75,1.25]$} & {$[1.25,3.25]$} & {$[3.25,4.75]$} \\
$M$ & 2 & 1 & 0 & 1 & 2 & 3 \\
'adjacencies' & $A 3, A 4$ & $A 4$ & NULL & $C 1$ & $C 1, C 2$ & $C 1, C 2, C 3$ \\
\hline
\end{tabular}

Table 8 The changing process of the number of 'adjacencies' of the desired SV " $X$ " in Category IV (i

\begin{tabular}{lllllll}
\hline$f_{D_{X}}, k H z$ & {$[-4.75,-3.25]$} & {$[-3.25,-2.75]$} & {$[-2.75,-0.75]$} & {$[-0.75,1.25]$} & {$[1.25,3.25]$} & {$[3.25,4.75]$} \\
$M$ & 1 & 0 & 1 & 2 & 3 & 4 \\
'adjacencies' & $A 4$ & NULL & $C 1$ & $C 1, C 2$ & $C 1, C 2, C 3$ & $C 1, C 2, C 3, C 4$ \\
\hline
\end{tabular}


As shown in Category $I$, it is obtained that $p_{v_{42}}=48.9 \%, \quad p_{v_{42}}=48.9 \%, \quad p_{v_{43}}=10.2 \% \quad$ and $p_{v_{44}}=0 \%$.

\subsection{A priori probability $p_{M_{k}}$}

The desired SV would locate in one of these four Categories. The priori probability $p_{M_{k}}$ is different when the desired SV lies in a different Category. In a specific Category,

- the first step is to figure out the Doppler interval when the desired SV has one 'adjacency' and to calculate the probability that the desired SV has a Doppler shift within this interval. This percentage leads to the value of $p_{M_{1}}$.

- The second step is to figure out the Doppler interval when the desired SV has two 'adjacencies' and to calculate the probability that the desired SV has a Doppler shift within this interval. This percentage leads to the value of $p_{M_{2}}$.

- The third step is to figure out the Doppler interval when the desired SV has three 'adjacencies' and to calculate the probability that the desired SV has a Doppler shift within this interval. This percentage leads to the value of $p_{M_{3}}$.

- The last step is to figure out the Doppler interval when the desired SV has four 'adjacencies' and to calculate the probability that the desired SV has a Doppler shift within this interval. This percentage leads to the value of $p_{M_{4}}$.

The details are presented as follows.

\subsubsection{Category I}

Looking into Table 5, there is one 'adjacency' when $f_{D_{X}} \in[0.75,2.75] \cup[3.25,4.75] \mathrm{kHz}$. By means of the PDF of the Doppler shift, its integration over the specified interval is obtained as $p_{M_{1}}=41.3 \%$. Similarly, there are two 'adjacencies' when $f_{D_{X}} \in[-1.25,0.75] \mathrm{kHz}$, $p_{M_{2}}=12.2 \%$. There are three 'adjacencies' when $p_{M_{3}}=14.5 \%, p_{M_{3}}=14.5 \%$. There are four 'adjacencies' when $f_{D_{X}} \in[-4.75,-3.25] \mathrm{kHz}, p_{M_{4}}=27.8 \%$.

\subsubsection{Category II}

Looking into Table 6, there is one 'adjacency' when $f_{D_{X}} \in[-1.25,0.75] \cup[1.25,3.25] \mathrm{kHz}$. Similar to Category I, we have $p_{M_{1}}=26.8 \%$. There are two 'adjacencies'

when

$p_{M_{2}}=42.3 \%, p_{M_{2}}=42.3 \%$. There are three 'adjacencies' when $f_{D_{X}} \in[-4.75,-3.25] \mathrm{kHz}, \quad p_{M_{3}}=27.8 \%$. Since there are at most three 'adjacencies' for SVs in this Category, $p_{M_{4}}=0 \%$.

\subsubsection{Category III}

Looking into Table 7, there is one 'adjacency' when $f_{D_{X}} \in[-3.25,-1.25] \cup[-0.75,1.25] \mathrm{kHz}$, we have
$p_{M_{2}}=42.3 \%$. There are two 'adjacencies' when $p_{M_{2}}=42.3 \%, p_{M_{2}}=42.3 \%$. There are three 'adjacencies' when $f_{D_{X}} \in[3.25,4.75] \mathrm{kHz}, p_{M_{3}}=27.8 \%$. Additionally, $p_{M_{4}}=0 \%$.

6.4.4 Category IV

Looking into Table 8, there is one 'adjacency' when $p_{M_{1}}=41.3 \%, p_{M_{1}}=41.3 \%$. There are two 'adjacencies' when $f_{D_{X}} \in[-0.75,1.25] \mathrm{kHz}, p_{M_{2}}=12.2 \%$. There are three 'adjacencies' when $f_{D_{X}} \in[1.25,3.25] \mathrm{kHz}$, $p_{M_{3}}=14.5 \%$. There are four 'adjacencies' when $p_{M_{3}}=27.8 \%, p_{M_{3}}=27.8 \%$.

\subsection{Conditional probability $p_{k}$ (collision $j$ visible)}

The conditional probability is related with the null-tonull bandwidth of each subcarrier, $w_{n n}$. The smaller the $\mathrm{OBI}$, the bigger the bandwidth, the larger the conditional probability. We consider two cases: (1) the OBI is equal to $1 \mathrm{~ms}$, i.e. the null-to-null bandwidth of each subcarrier is equal to $2 \mathrm{kHz}$; and (2) the $\mathrm{OBI}$ is equal to $20 \mathrm{~ms}$, i.e. the null-to-null bandwidth of each subcarrier is equal to $0.1 \mathrm{kHz}$.

The desired SV would locate in one of these four Categories. In each Catogory, in order to obtain the conditional probability $p_{k}$ (collision $\mid j$ visible), the first step is to determine the 'adjacencies' of the desired SV and the corresponding Doppler shifts when any of them is visible. Assuming the center frequency of the desired SV without Doppler shift is at zero, the center frequency of the desired SV after considering Doppler shift can be obtained. Furthermore, the center frequency of the 'adjacencies' of the desired SV safter considering Doppler shift can be obtained based on the subcarrier space of the

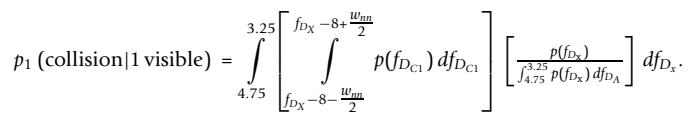

transmitting signal of the 'adjacencies' and the desired SV. The POC at a specific Doppler bin of the desired SV can then be calculated based on the non-uniform distribution of the Doppler shift. Finally, based on this POC at a specific Doppler bin, the conditional probability $p_{k}$ (collision $\mid j$ visible) can be obtained. The details are presented as follows.

\subsubsection{Category}

When $f_{D_{X}} \in[3.25,4.75] \mathrm{kHz}, C 1$ is the unique 'adjacency' of B1. Referring to Table 3, the Doppler shift on the visible $C 1$ belongs to the interval $[-4.75,4.75] \mathrm{kHz}$. So, relative to $B 1$ without Doppler, the shifted $C 1$ lies in [8 $-4.75,8+4.75] \mathrm{kHz}$ since the subcarrier spacing between $C 1$ and $B 1$ is $8 \mathrm{kHz}$. The shifted $B 1$ obviously lies in [3.25, $4.75] \mathrm{kHz}$. In other words, wherever $B 1$ shifts, the collision 
with $C 1$ cannot be avoided, and the magnitude of the collision is determined by $w_{n n}$. At each $f_{D_{X}}$, the POC is obtained as $\int_{f_{D_{X}}-8-\frac{w_{n n}}{2}}^{f_{D_{X}}-8+\frac{w_{n n}}{2}} p\left(f_{D_{C 1}}\right) d f_{D_{C 1}}$, where $f_{D_{C 1}}$ is the PDF of the Doppler shift on the 'adjacency' $\mathrm{C} 1$. However, a different $f_{D_{X}}$ corresponds to a different POC due to the non-uniform distribution of the Doppler shift. Based on the distribution of $f_{D_{X}}$, denoted by $p\left(f_{D_{X}}\right)$, we have

$$
\begin{aligned}
& p_{1}\left(\text { collision } \mid 1 \text { visible, } f_{D_{X}} \in[3.25,4.75]\right) \\
= & \int_{f_{D_{X}}-8-\frac{w_{n n}}{2}}^{f_{D_{X}}-8+\frac{w_{n n}}{2}} p\left(f_{D_{C 1}}\right) d f_{D_{C 1}},
\end{aligned}
$$

and

$$
p\left(f_{D_{X}} \mid f_{D_{X}} \in[3.25,4.75]\right)=\frac{p\left(f_{D_{X}}\right)}{\int_{4.75}^{3.25} p\left(f_{D_{X}}\right) d f_{D_{X}}} .
$$

So, the average POC when $f_{D_{X}} \in[3.25,4.75] \mathrm{kHz}$ can be expressed as Equation (18). Based on (18), and on Figures 8, we have $p_{1}($ collision $\mid 1$ visible $)=\{24.3 \%$, $1.42 \%\}^{\mathrm{g}}$
When $f_{D_{X}} \in[-1.25,0.75] \mathrm{kHz}$, there are two 'adjacencies': $A 3$ and $A 4$. If $A 3$ is visible while $A 4$ is not, looking into Table 3, the Doppler shift on $A 3$ is in $[-4.75,4,43]$ $\mathrm{kHz}$ and so, relative to $B 1, A 3$ lies in $[-8.75,0.43] \mathrm{kHz}$. When $f_{D_{X}} \in[-1.25,0.43] \mathrm{kHz}$, a collision occurs in the same way as above. In this case, the POC is obtained as $\{27.3 \%, 1.28 \%\}$. Thus, the integrated POC is $\{22.9 \%$, $1.08 \%$. If $A 4$ is visible while $A 3$ is not, looking into Table 3, the Doppler shift on $A 4$ is in $[-4.43,4,75] \mathrm{kHz}$ and so, relative to $B 1, A 4$ lies in $[-6.73,2.75] \mathrm{kHz}$ and the POC is approximately $\{15.8 \%, 0.78 \%\}$. Considering the relative visibility range, the resulting conditional POC for one visible 'adjacency' is $p_{2}$ (collision $\mid 1$ visible $)=\{19.4 \%$, $0.92 \%$ \}. If both $A 3$ and $A 4$ are visible, they lie respectively in $[0.43,0.75]$ and $[-6.75,-6.43] \mathrm{kHz}$ relative to $B 1$. It is evident that $B 1$ may collide with $A 3$ while not with $A 4$. When $f_{D_{X}} \in[-1.25,0.43] \mathrm{kHz}$, no collision occurs. When $f_{D_{X}} \in[0.43,0.75] \mathrm{kHz}$, the collision occurs with the following probability: $\{100 \%, 26.8 \%\}$. Finally, the integrated conditional POC for two visible 'adjacencies' is $p_{2}$ $($ collision $\mid 2$ visible $)=\{29.8 \%, 4.20 \%\}$.

When $f_{D_{X}} \in[-3.25,-1.25] \mathrm{kHz}$, there are three 'adjacencies': $A 2, A 3$ and $A 4$. If exactly $A 2$ is visible, it lies in $[-7.98,1.57] \mathrm{kHz}$, the corresponding POC is $\{40.6 \%$,

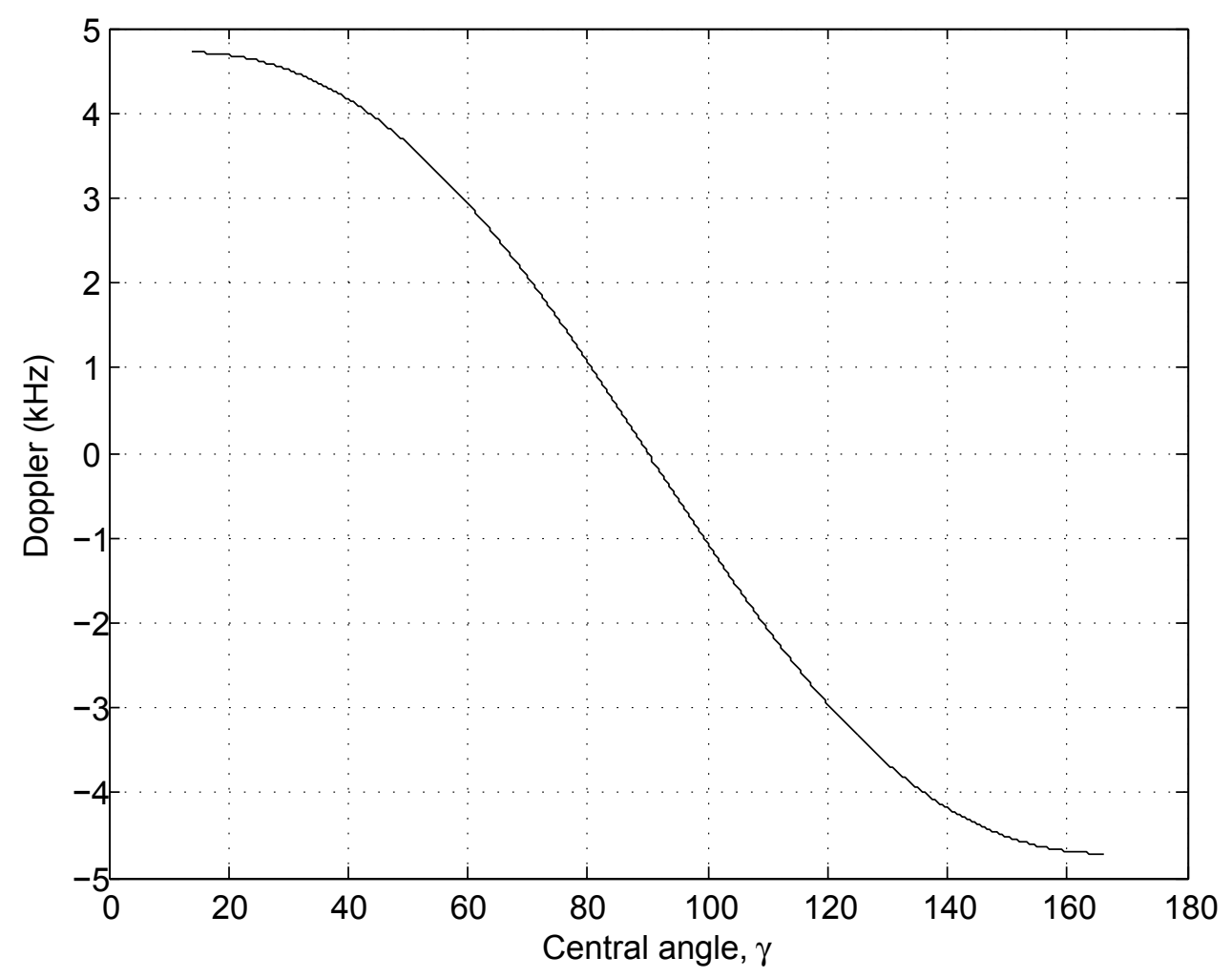

Figure 8 Doppler shift $f_{D}$ for a stationary in-plane receiver, as a function of $\gamma$. 
$1.90 \%\}$. If exactly $A 3$ is visible, it lies in $[-8.43,0.43] \mathrm{kHz}$, and the POC is $\{18.5 \%, 0.91 \%\}$. If exactly $A 4$ is visible, $A 4$ lies in $[-6.43,-0.02] \mathrm{kHz}$, and the corresponding POC is $\{25.1 \%, 1.24 \%\}$. Taking the visibility range into consideration, the conditional POC for one visible 'adjacency' is $p_{3}$ (collision 11 visible $)=\{26.8 \%, 1.29 \%\}$. When $A 2$ and $A 3$ are visible while $A 4$ is not, $A 2$ lies in $[-1.57,-1.25] \mathrm{kHz}$, which leads to the integrated POC $\{26.0 \%, 3.75 \%\}$. Any other combinations of two visible 'adjacencies' cannot cause collision. Taking the visibility range into consideration, $p_{3}($ collision $\mid 2$ visible $)=\{5.9 \%, 0.8 \%\}$. Additionally, since the condition of three simultaneously visible 'adjacencies' is false, calculating the POC under this condition is meaningless because its contribution to the collision is 0 when removing the condition.

When $f_{D_{X}} \in[-4.75,-3.25] \mathrm{kHz}$, there are four 'adjacencies': $A 1, A 2, A 3$ and $A 4$. When exactly one is visible, $A 2$ and $A 3$ lie in $[-7.98,-1.57]$ and $[-8.43,-2.02] \mathrm{kHz}$, respectively. So, we have $p_{4}$ (collision $\mid 1$ visible $)=\{26.3 \%$, $1.29 \%\}$. The scenarios of two visible 'adjacencies' cannot cause collision except for $A 1$ and $A 4$, where $A 1$ lies in $[-9.98,-3.57] \mathrm{kHz}$, leading to a collision probability of $\{43.9 \%, 2.00 \%\}$, and $A 4$ lies in $[-6.43,-0.02] \mathrm{kHz}$, leading to a collision probability of $\{29.9 \%, 1.46 \%\}$. Since no collision occurs between SVs in one orbit, when $A 1$ collides with $B 1$, $A 4$ doesn't. Also, when $A 4$ collides with $B 1, A 1$ doesn't. So the POC for such scenario is $\{73.8 \%, 3.46 \%\}$. Averaged by the visible range, $p_{4}$ (collision $\mid 2$ visible $)=\{35.4 \%, 1.66 \%\}$. For the scenario of three visible 'adjacencies' of $A 1, A 2$ and $A 4, A 1$ lies in $[-3.57,-3.25] \mathrm{kHz}$, which provides a collision probability of $\{20.5 \%, 2.90 \%\}$, while the scenario of $A 1, A 3$ and $A 4$ cannot cause collision. So, we have $p_{4}$ (collision $\mid 3$ visible $)=\{10.2 \%, 1.45 \%\}$. Additionally, the condition of four simultaneously visible 'adjacencies' is false and its contribution to the collision is 0 when removing the condition.

\subsubsection{Category II}

When $f_{D_{X}} \in[-1.25,-0.75] \mathrm{kHz}$, there is one 'adjacency' $A 4$, and the corresponding POC is equal to $\{23.8 \%, 1.57 \%\}$. When $f_{D_{X}} \in[1.25,3.25] \mathrm{kHz}$, there is one 'adjacency' $C 1$, and the POC is $\{23.5 \%, 1.49 \%\}$. Considering the occurrence probability, 12.2 and $14.5 \%$ for $f_{D_{X}} \in[-1.25,-0.75] \mathrm{kHz}$ and $f_{D_{X}} \in[1.25,3.25] \mathrm{kHz}$, respectively, the average conditional POC is obtained as $p_{1}($ collision $\mid 1$ visible $)=\{23.6 \%, 1.53 \%\}$.

When $f_{D_{X}} \in[3.25,4.75] \mathrm{kHz}$, there are two 'adjacencies': $C 1$ and $C 2$. When only $C 1$ is visible, it lies in [1.25, $10.43] \mathrm{kHz}$ relative to $B 2$, and the corresponding POC is $\{15.7 \%, 0.77 \%\}$. When only $C 2$ is visible, it lies in [3.57, 12.57] $\mathrm{kHz}$ relative to $B 2$, and the corresponding POC is $\{24.8 \%, 1.15 \%\}$. Taking the visibility range into consideration, the resulting POC for one visible 'adjacency' is $\{20.3 \%$, $0.96 \%$.
When both $C 1$ and $C 2$ are visible, $C 2$ lies in [3.25, 3.57] $\mathrm{kHz}$, the corresponding POC is $\{20.5 \%, 3.00 \%\}$, while $C 1$ cannot cause any collisions. So, the resulting conditional POC for two visible 'adjacencies' is $\{20.5 \%, 3.00 \%\}$. On the other hand, when $f_{D_{X}} \in[-3.25,-1.25] \mathrm{kHz}$, there are two 'adjacencies': $A 3$ and $A 4$, and the POC is $\{19.4 \%$, $0.92 \%\}$ for one visible 'adjacency' and $\{26.0 \%, 3.75 \%\}$ for two simultaneously visible 'adjacencies'. Also, the occurrence probability for $f_{D_{X}} \in[3.25,4.75] \mathrm{kHz}$ and $f_{D_{X}} \in[-3.25,-1.25] \mathrm{kHz}$ is 27.8 and $14.5 \%$, respectively. Overall, $p_{2}$ (collision $\mid 1$ visible $)=\{20.0 \%, 0.95 \%\}$. and $p_{2}$ $($ collision $\mid 2$ visible $)=\{22.5 \%, 3.26 \%\}$.

When $f_{D_{X}} \in[-4.75,-3.25] \mathrm{kHz}$, there are three 'adjacencies': $A 2, A 3$ and $A 4$. When only $A 2$ is visible, the POC is $\{43.9 \%, 2.00 \%\}$. When only $A 3$ is visible, the POC is $\{18.5 \%, 0.91 \%\}$. When only $A 4$ is visible, the POC is $\{24.9 \%, 1.23 \%\}$. Thus, we have $p_{3}$ (collision 1 visible $)=\{27.6 \%, 1.32 \%\}$. When $A 2$ and $A 3$ are visible while $A 4$ is not, the POC is $\{20.5 \%, 2.90 \%\}$, while other combinations of two SV's being visible cannot lead to a collision. Thus, $p_{3}($ collision $\mid 2$ visible $)=\{4.5 \%, 0.62 \%\}$. Additionally, the condition of three simultaneously visible 'adjacencies' is false, so its contribution to the POC is 0 when removing this condition.

Since the condition of four 'adjacencies' is also false, its contribution to the $\mathrm{POC}$ is also 0 when removing this condition.

\subsubsection{Category III}

When $f_{D_{X}} \in[-3.25,-1.25] \mathrm{kHz}$, there is one 'adjacency' $A 4$, and the corresponding POC is equal to $\{23.5 \%, 1.49 \%\}$. When $f_{D_{X}} \in[-0.75,1.25] \mathrm{kHz}$, there is one 'adjacency' $C 1$, and the POC is $\{23.8 \%, 1.57 \%\}$. Also, the occurrence probability for $f_{D_{X}} \in[-3.25,-1.25] \mathrm{kHz}$ and $f_{D_{X}} \in[-0.75,1.25] \mathrm{kHz}$ is 14.5 and $12.2 \%$, respectively. So, the average conditional POC is $p_{1}$ (collision $\mid 1$ visible $)=\{23.6 \%, 1.53 \%\}$.

When $f_{D_{X}} \in[-4.75,-3.25] \mathrm{kHz}$, there are two 'adjacencies': $A 3$ and $A 4$. When only $A 3$ is visible, the POC is $\{24.9 \%, 1.13 \%\}$. When only $A 4$ is visible, it is $\{15.7 \%$, $0.77 \%\}$. So, the average value is $\{20.3 \%, 0.95 \%\}$. When both $A 3$ and $A 4$ are visible, the POC is $\{20.5 \%, 3.00 \%\}$. When $f_{D_{X}} \in[1.25,3.25] \mathrm{kHz}$, there are two 'adjacencies': $C 1$ and $C 2$. When only $C 1$ is visible, the POC is $\{15.7 \%, 0.77 \%\}$. When only $C 2$ is visible, it is $\{24.1 \%$, $1.13 \%\}$. Thus, taking the visible range into consideration, we have $\{19.9 \%, 0.95 \%\}$. When both $C 1$ and $C 2$ are visible, the POC is $\{20.5 \%, 3.15 \%\}$. Also, the occurrence probability for $f_{D_{X}} \in[-4.75,-3.25] \mathrm{kHz}$ and $f_{D_{X}} \in[1.25,3.25] \mathrm{kHz}$ is 27.8 and $14.5 \%$, respectively. Overall, the conditional POC is $p_{2}$ (collision $\mid 1$ visible) $=$ $\{20.2 \%, 0.95 \%\}$ for one visible 'adjacency' and $p_{2}$ 
$($ collision $\mid 2$ visible $)=\{20.5 \%, 3.05 \%\}$ for two visible 'adjacencies'.

When $f_{D_{X}} \in[3.25,4.75] \mathrm{kHz}$, there are three 'adjacencies': $C 1, C 2$ and $C 3$. When only $C 1$ is visible, $C 1$ lies in $[2.02,8.43] \mathrm{kHz}$ relative to $B 3$, and the POC is $\{32.9 \%$, $1.63 \%\}$. When only $C 2$ is visible, $C 2$ lies in $[1.57,10.43]$ $\mathrm{kHz}$, and the POC is $\{33.9 \%, 1.66 \%\}$. When only $\mathrm{C} 3$ is visible, C3 lies in $[3.57,9.98] \mathrm{kHz}$, and the POC is $\{48.2 \%$, $2.37 \%$. Taking the visibility range into consideration, the corresponding conditional POC can be obtained as $p_{3}$ $($ collision $\mid 1$ visible $)=\{37.6 \%, 1.85 \%\}$. The scenarios of two visible 'adjacencies' cannot cause any collisions except for $C 2$ and $C 3$, where $C 3$ lies in $[-4.75,-4.43] \mathrm{kHz}$. This leads to a POC $\{20.5 \%, 3.12 \%\}$. Taking the visibility range into consideration, we have $p_{3}($ collision $\mid 2$ visible $)=\{4.5 \%$, $0.67 \%$. Additionally, the condition of three simultaneously visible 'adjacencies' is false, so its contribution to the POC is 0 when removing this condition.

Since the condition of having four 'adjacencies' is false, its contribution to the $\mathrm{POC}$ is 0 when removing this condition.

\subsubsection{Category IV}

When $f_{D_{X}} \in[-4.75,-3.25] \mathrm{kHz}$, there is one 'adjacency' $A 4$, and the corresponding POC is equal to $\{24.3 \%, 1.42 \%\}$. When $f_{D_{X}} \in[-2.75,-0.75] \mathrm{kHz}$, there is one 'adjacency' $C 1$, and the POC is $\{24.1 \%, 1.64 \%\}$. Also, the occurrence probability for $f_{D_{X}} \in[-4.75,-3.25] \mathrm{kHz}$ and $f_{D_{X}} \in[-2.75,-0.75] \mathrm{kHz}$ is 27.8 and $13.5 \%$, respectively. Similarly to the above, the conditional POC is $p_{1}$ $($ collision $\mid 1$ visible $)=\{24.2 \%, 1.50 \%\}$.

When $f_{D_{X}} \in[-0.75,1.25] \mathrm{kHz}$, there are two 'adjacencies': $C 1$ and $C 2$. When only $C 1$ is visible, the POC is $\{15.8 \%, 0.78 \%\}$. When only $C 2$ is visible, it is $\{23.1 \%$, $1.10 \%\}$, respectively. Thus, taking the visible range into consideration, we have $\{19.4 \%, 0.94 \%\}$. When both $C 1$ and $C 2$ are visible, the POC is $\{16.0 \%, 4.61 \%\}$. Similarly, we have $p_{2}$ (collision $\mid 1$ visible $)=\{19.4 \%, 0.94 \%]$ and $p_{2}$ $($ collision $\mid 2$ visible $)=\{16.0 \%, 4.61 \%\}$.

When $f_{D_{X}} \in[1.25,3.25] \mathrm{kHz}$, there are three 'adjacencies': $C 1, C 2$ and $C 3$. When only $C 1$ is visible, $C 1$ lies in $[0.02,6.43] \mathrm{kHz}$ relative to $B 4$, and the POC is $\{25.1 \%, 1.24 \%\}$. When only $C 2$ is visible, $C 2$ lies in $[-0.43,8.43] \mathrm{kHz}$, and the POC is $\{18.5 \%, 0.91 \%\}$. When only $C 3$ is visible, $C 3$ lies in $[1.57,7.98] \mathrm{kHz}$, and the POC is $\{41.0 \%, 1.94 \%\}$. Taking the visibility range into consideration, the conditional POC is $p_{3}$ (collision $\mid 1$ visible $)=\{26.9 \%, 1.30 \%\}$. The scenarios of two visible 'adjacencies' cannot cause any collisions except for $C 2$ and $C 3$, where $C 3$ lies in $[1.25,1.57] \mathrm{kHz}$. This leads to a POC $\{26.0 \%, 4.02 \%\}$. Taking the visible range into consideration, $p_{3}($ collision $\mid 2$ visible $)=\{9.1 \%, 1.42 \%\}$. Additionally, the condition of three simultaneously visible 'adjacencies' in this case is false and its contribution to the POC is equal to 0 when removing this condition.

When $f_{D_{X}} \in[3.25,4.75] \mathrm{kHz}$, there are four 'adjacencies': $C 1, C 2, C 3$ and $C 4$. When exactly one is visible, $C 2$ and $C 3$ respectively lie in [2.02, 8.43] and [1.57, 7.98] $\mathrm{kHz}$. So, we have $p_{4}$ (collision $\mid 1$ visible $)=\{26.3 \%$, $1.30 \%$ \}. The scenarios of two visible 'adjacencies' cannot cause any collisions except for $C 1$ and $C 4$, where $C 1$ lies in $[-1.98,4.43] \mathrm{kHz}$. This leads to a POC $\{30.0 \%, 1.46 \%\}$, respectively. Taking the visible range into consideration, we have $p_{4}($ collision $\mid 2$ visible $)=\{14.4 \%, 0.70 \%\}$. For the scenario of three visible 'adjacencies' of $C 1, C 3$ and $C 4$, $C 4$ lies in $[3.25,3.57] \mathrm{kHz}$, which provides a POC $\{20.5 \%, 3.12 \%\}$, while the scenario of $C 1, C 2$ and $C 4$ cannot cause any collisions. So, $p_{4}$ (collision $\mid 3$ visible $)=$ $\{10.3 \%, 1.56 \%\}$. Additionally, the condition of four simultaneously visible 'adjacencies' is false and its contribution to the POC is 0 when removing this condition.

\subsection{Probability of collision}

6.6.1 Observation interval: $1 \mathrm{~ms}$

For $6 \mathrm{SVs}$ in Category I, the a priori probabilities are $p_{c_{2}}=15.9 \%, \quad p_{c_{2}}=15.9 \%, \quad p_{c_{3}}=20.7 \%, \quad$ and $p_{c_{4}}=28.9 \%$, respectively. The POC for Category I is therefore obtained as $p_{c}^{I}=\sum_{k=1}^{4}\left(p_{c_{k}} p_{\mathrm{M}_{k}}\right)=17.2 \%$. Similarly, for 6 SVs in Category II, the a priori probabilities are $p_{c_{1}}=9.96 \%, p_{c_{2}}=16.0 \%, p_{c_{3}}=24.5 \%$, and $p_{c_{4}}=0 \%$, respectively. The POC for Category II is therefore obtained as $P_{c}^{I I}=\sum_{k=1}^{4}\left(p_{c_{k}} p_{M_{k}}\right)=15.3 \%$. For 6 SVs in Category III, the a priori probabilities are $p_{c_{2}}=16.0 \%, \quad p_{c_{2}}=16.0 \% p_{c_{3}}=28.2 \%$, and $p_{c_{4}}=0 \%$ respectively. The POG for Category III is therefore obtained as $p_{c}^{I I I}=\sum_{k=1}^{4}\left(p_{c_{k}} p_{M_{k}}\right)=17.3 \%$. For $6 \mathrm{SVs}$ in Category IV, the a priori probabilities are $p_{c_{2}}=15.2 \%, \quad p_{c_{2}}=15.2 \%, \quad p_{c_{3}}=21.6 \%, \quad$ and $p_{c_{4}}=18.7 \%$, respectively. The POC for Category IV is therefore obtained as $p_{c}^{I V}=\sum_{k=1}^{4}\left(p_{c_{k}} p_{M_{k}}\right)=14.4 \%$.

The overall POC can therefore be obtained by taking the average of $p_{c}^{I}, p_{c}^{I I}, p_{c}^{I I I}$ and $p_{c}^{I V}$ since they are equally probable:

$$
p_{c}=\frac{p_{c}^{I}+p_{c}^{I I}+p_{c}^{I I I}+p_{c}^{I V}}{4}=16.1 \%
$$

Without loss of generality, assuming that there are eight visible SVs for a certain GPS receiver, the probability that there exist at most 3 visible non-collision SVs is

$$
P_{p c}=\sum_{k=0}^{3} C_{8}^{k}\left(1-p_{c}\right)^{k} p_{c}^{8-k}=0.39 \%
$$


where $C_{8}^{k}$ denotes the number of combinations of choosing $k$ out of eight SVs. Therefore, the probability that there exist at least four visible non-collision SVs can be given by

$$
P_{s}=1-P_{p c}=99.61 \% .
$$

In other words, the probability for successful positioning is equal to $99.61 \%$.

\subsubsection{Observation interval: $20 \mathrm{~ms}$}

By following a similar analysis to the previous section, one can show that it is obtained that $p_{c}^{I}=0.93 \%, p_{c}^{I I}=0.85 \%, p_{c}^{I I I}=0.95 \%$, and $p_{c}^{I V}=0.84 \%$, and that, the overall POC $p_{c}=0.89 \%$. Once again, assuming that there are eight visible SVs for a certain GPS receiver, the probability that there exist at most three visible non-collision SVs is $P_{p c} \approx 3 \times 10^{-9}$. Therefore, the probability that there exist at least four visible non-collision SVs is

$$
P_{s}=1-P_{p c} \approx 100 \% .
$$

In other words, the probability for successful positioning is approximately equal to $100 \%$.

\section{Numerical results}

In this section, the theory proposed above is demonstrated by numerical simulations. To verify the SNR gain, Two signals are used for demonstration: a MC code defined by (1) and (2) and the C/A code, where the MC code contains $N=1024$ subcarrier slots and one period of the C/A code contains 1, 023 chips. Both signals have an equal energy $\xi$ and an equal null-to-null bandwidth $2 B=2.048 \mathrm{MHz}$. The signal duration, $T_{s}$, is selected as $1.023 \mathrm{~ms}$ for both signals.

To test the effect of NBI and Doppler shift to the reception of the proposed $\mathrm{MC}$ codes, a computer orbit generation program is employed. In this orbit generation program, the configuration for the numerical results consists of 24 SVs in six circular orbits (eccentricity 0 ), four SVs in each circular orbit arranging as Figure 6. The six planes are set to have a uniformly $55^{\circ}$ inclination (tilt relative to Earth's equator) and to be separated by $60^{\circ}$ right ascension of the ascending node (angle along the equator from a reference point to the orbit's intersection). And, the orbit altitude is set as 20, 200 $\mathrm{km}$. The GPS receiver in our computer program is located at Calgary, Canada $\left(51^{\circ} \mathrm{N}\right.$ and $\left.114^{\circ} \mathrm{W}\right)$, Washington, DC $\left(39^{\circ} \mathrm{N}\right.$ and $\left.77^{\circ} \mathrm{W}\right)$, London, UK $\left(51^{\circ} \mathrm{N}\right.$ and $\left.0^{\circ} \mathrm{W}\right)$ and Melbourne, Australia $\left(37^{\circ} \mathrm{S}\right.$ and $\left.144^{\circ} \mathrm{E}\right)$, respectively.

\subsection{SNR gain}

Figure 9 compares the $\mathrm{MC}$ code with the $\mathrm{C} / \mathrm{A}$ code for range estimation in terms of their CRLB and their maximum likelihood estimators (MLE) by displaying the root mean square error (RMSE) in meter versus SNR in order to demonstrate the proposed theory in (7). Consistent with the theoretical analysis, for a fixed ranging accuracy, the required SNR for the MC code is lower than that for the C/A code by approximately $4.73 \mathrm{~dB}$, indicating a $4.73 \mathrm{~dB}$ SNR gain using the proposed $\mathrm{MC}$ code compared to the current C/A code. This SNR gain is significant for GPS downtown positioning, tunnel positioning and even indoor positioning where the current GPS signal is too weak to detect. This result demonstrates that approximation $(a)$ used in (4)-(7) is a good approximation when $N=1024$. Furthermore, Figure 9 shows that the MLE attains the corresponding CRLB at most SNR values in the asymptotic region [22]. One note to be pointed out that the MLE at certain SNR looks lower than its corresponding CRLB because the iteration in Monte Carlo simulation is not sufficient. In theory, the MLE at all SNR values in the asymptotic region must exactly attain its corresponding CRLB.

\subsection{Robustness against NBI}

Figure 10 shows the simulation result for the probability of successful positioning $P_{s}$ versus $M$ NBIs, where the proposed $\mathrm{MC}$ code is used for $24 \mathrm{SVs}, M=1,2, \ldots, 8$ and each NBI is referred as a frequency tone [21]. This statistic is an average value of all measures in approximately $12 \mathrm{~h}$, where each observation duration is $1 \mathrm{~ms}$ while the interval between two observations is $1 \mathrm{~s}$. One can see that four numerical results in Figure 10, corresponding to GPS receivers at four different locations, are all approximately consistent with the proposed theory in (10), which have a similar performance against NBIs. One can also see that, even with 8 existing NBIs, the probability of successful positioning is still above $95 \%$, indicating its robustness against NBI.

\subsection{Probability for successful positioning under Doppler effects}

Figures 11 and 12 show simulation results of the probability of successful positioning $P_{s}$ versus the SV ID $S_{I D}$. Even though the GPS receiver at each of four location can only simultaneously see 4 to $12 \mathrm{SVs}$, it can communicate with all 24 SVs when considering all the observation time. The OBI is selected as $1 \mathrm{~ms}$ in Figure 11 and $20 \mathrm{~ms}$ in Figure 12, respectively. It is noticed that all four GPS receivers at four different locations have a similar performance against Doppler shift. Furthermore, all 24 SVs also have a similar probability of successful positioning for both cases. The average probability of successful positioning for $24 \mathrm{SVs}$, seen from Figure 11, is approximately $99.58 \%$, equal to the theoretical value of $99.61 \%$ as given in (21). For the case of $20 \mathrm{~ms}$ OBI, the average probability of successful positioning for 24 


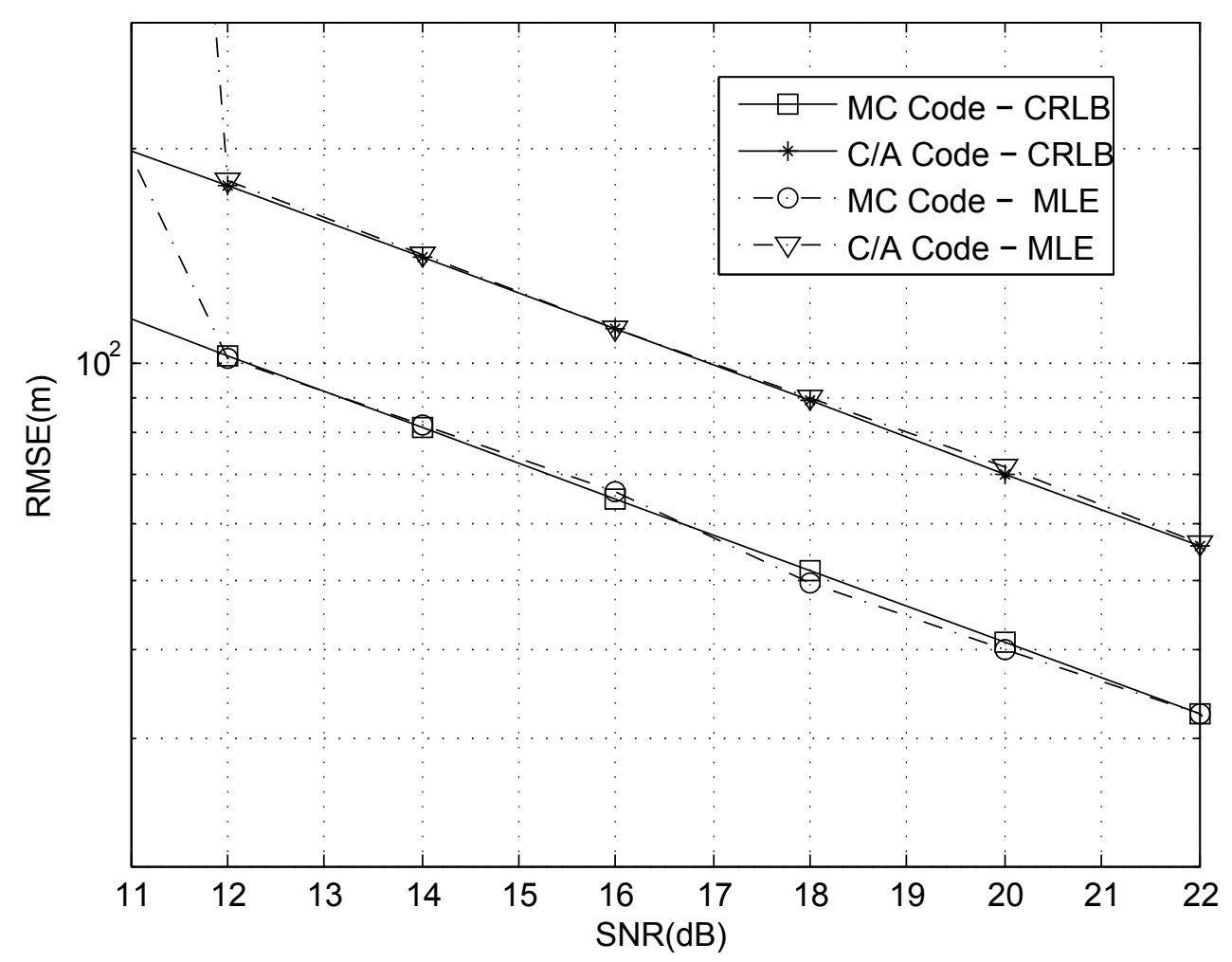

Figure 9 CRLB and MLE for range estimation for the proposed MC code and the C/A code.

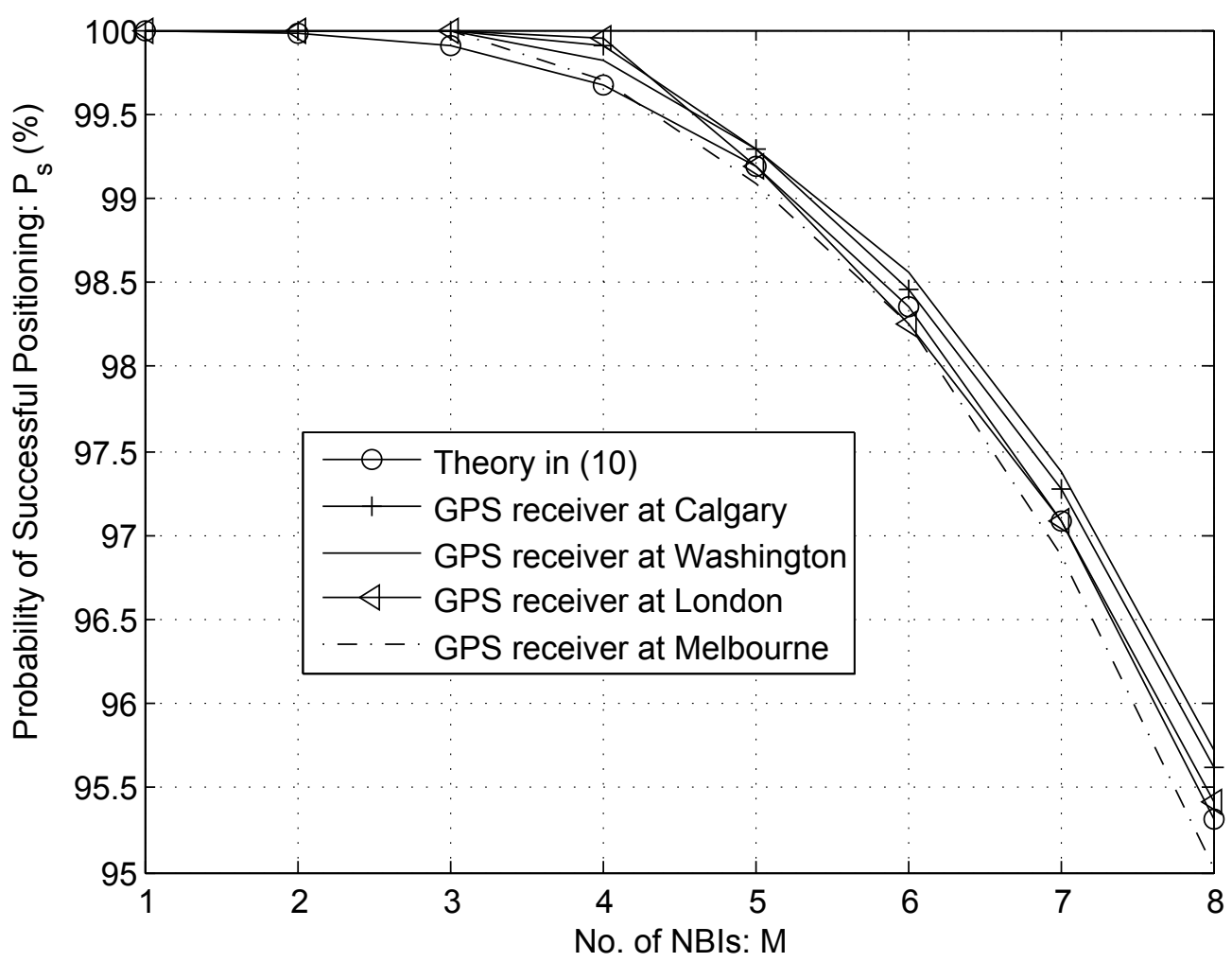

Figure 10 Probability of successful positioning, $P_{s}$, under multiple NBIs, $M$. 


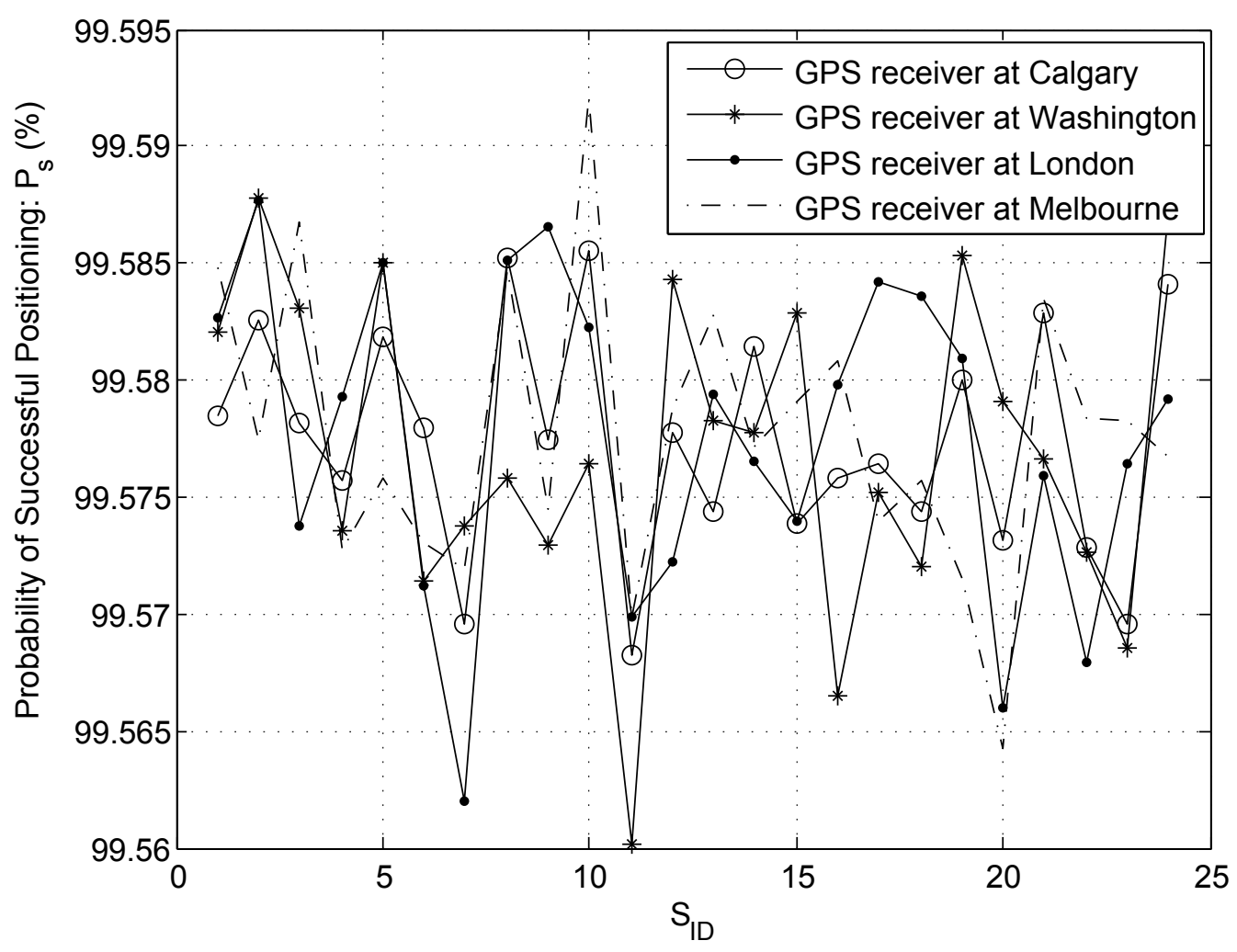

Figure 11 Probability of successful positioning, $P_{s}$, under Doppler effects, where the observation interval is selected as $1 \mathrm{~ms}$.

SVs, seen from Figure 12, is approximately 99.9996\%, equal to the theoretical value of $100 \%$ as given in (22).

\section{Conclusion and future work}

The unfiltered MC modulation has been proposed as an alternative for the GPS C/A code in this article. As per our arrangement, each SV takes up only 42 uniformly spaced subcarrier slots while approximately occupying the same bandwidth as the C/A code in GPS. In this way, the proposed MC code was shown to attain a 4.73 $\mathrm{dB}$ SNR gain in terms of range estimation compared to the current C/A code. This $4.73 \mathrm{~dB}$ SNR gain is significant and possibly helpful for GPS downtown's positioning, tunnel's positioning and even indoor positioning. The transmission and reception of the proposed MC code for the next-generation GNSS are provided in detail, and the range estimation using the proposed $\mathrm{MC}$ code is explained. Furthermore, the proposed MC code was shown to be robust against NBI by considering four GPS receivers at different locations, which is also one of the current GPS's disturbing things to overcome. Moreover, the POC and probability of successful positioning under Doppler shifts are thoroughly analyzed in theory and justified by computer simulations. It was shown that the Doppler shift has a negligible effect on the probability of successful positioning when using the proposed MC code as the GPS probing signal. To sum up, from the authors' best knowledge, the proposed MC code could be regarded as an available probing signal for the future next-generation GPS.

However, the dangerous adjacencies, affecting the POC value under Doppler shift, do depend on the strategy adopted to assign the set of subcarriers to the SVs. Our proposed arrangement results in no collision between groups of MC codes that are transmitted by SVs in one orbit. But we have no idea whether it is the best arrangement or not in terms of POC. The future work will focus on the optimization of the subcarrier arrangement for SVs.

\section{Appendix 1}

\section{Doppler shift for an in-plane GPS receiver}

Without loss of generality, let us assume that a GPS receiver resides on the surface of the earth at a point $R$ with $2 \mathrm{D}$ cartesian coordinates $(0, r)$ as shown in Figure 13a, where $r$ is the radius of the earth, and SV rotates around the earth in the XY-plane. In this case, the Doppler shift due to the SV motion is given by

$$
f_{D}=\frac{v}{c} f_{L 1} \cos \theta,
$$




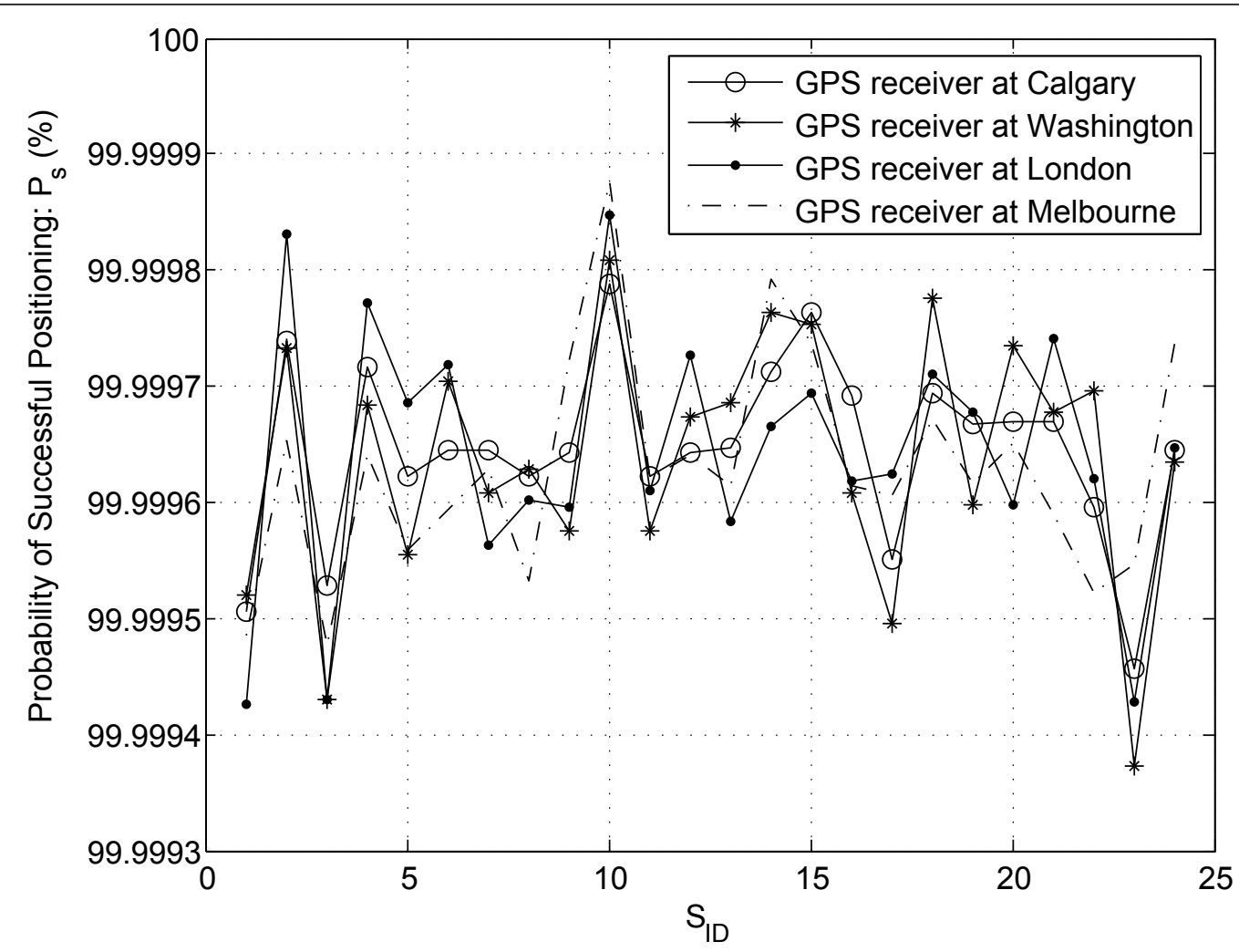

Figure 12 Probability of successful positioning, $P_{s}$, under Doppler effects, where the observation interval is selected as $20 \mathrm{~ms}$

where $v$ is the speed of SV, i.e. $3.874 \mathrm{~km} / \mathrm{s} ; c$ is the speed of light; $f_{L 1}$ is the carrier frequency of the coarse acquisition (CA) code, i.e. $1575.42 \mathrm{MHz}$ [12]; and $\theta$ is the angle between SV's forward velocity and the line of sight from SV to the receiver as shown in Figure 13a. Consider the maximum Doppler shift case, which happens at a point $S$ when SV ascends past the horizon of the receiver. In this case, the radial velocity at $R$ is 0.9

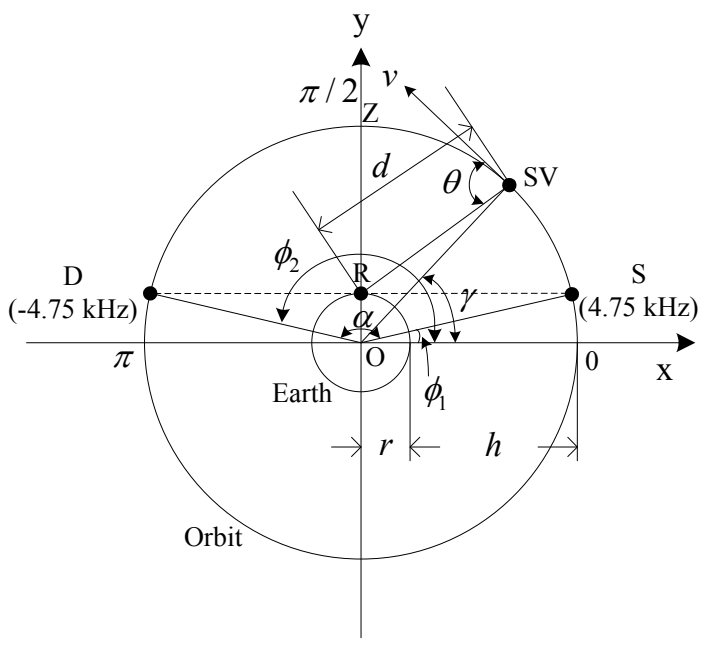

(a)

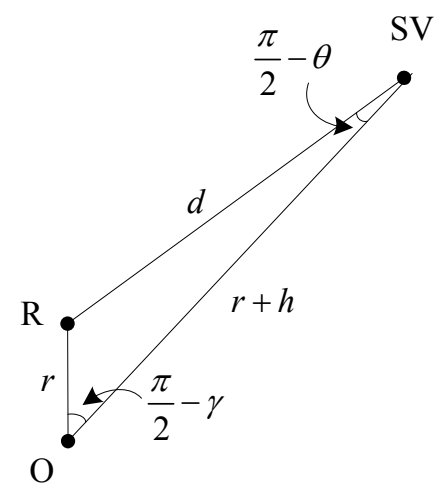

(b)

Figure 13 Visible range of an in-plane GPS receiver in terms of one SV (a) The visible region for a stationary in-plane GPS receiver, and (b) the triangle $\triangle O R(S V)$. 
$\mathrm{km} / \mathrm{s}$ [23] and the resulting maximum Doppler shift is $4.726 \mathrm{kHz}$. Similarly, the minimum Doppler shift is $-4.726 \mathrm{kHz}$ which occurs at a point $\mathrm{D}$ when SV descends past the horizon of the receiver. It is also interesting to note that $\theta$ changes from $76.5^{\circ}$ when ascending past the horizon at point $\mathrm{S}$ to $103.5^{\circ}$ when descending past the horizon at point $\mathrm{D}$.

Since the angle, $\varphi_{1}$, corresponding to the ascending node $S$, is $13.5^{\circ}$ and the angle, $\varphi_{2}$, corresponding to the descending node $\mathrm{D}$, is $166.5^{\circ}$, as shown in Figure 13a, the angle, $\alpha$, corresponding to the arc that is visible to $R$ in the orbital plane of SV, is $153^{\circ}$. In Figure 13b, the triangle $\triangle \mathrm{OR}(\mathrm{SV})$ is used to obtain the distance, $d$, between $\mathrm{SV}$ and the receiver, as

$$
d=\sqrt{(r+h)^{2}+r^{2}-2 r(r+h) \sin \gamma},
$$

where $r$ is the radius of the earth; $h$ is the height of $\mathrm{SV}$ from the earth, and $\gamma$ is referred to as the central angle of SV which is $\in\left[13.5^{\circ}, 166.5^{\circ}\right]$ in the visible region to $R$. Based on (24) and from Figure 13b, one can obtain the angle $\theta$ from

$$
\sin \theta=\frac{d^{2}+(r+h)^{2}-r^{2}}{2 d(r+h)} .
$$

In other words, the Doppler shift can be expressed as a function of $\gamma$ as follows

$$
f_{D}=\left\{\begin{array}{c}
\frac{v}{c} f_{L 1} \sqrt{1-\left[\frac{r(1-\sin \gamma)+h}{\sqrt{(r+h)^{2}+r^{2}-2 r(r+h) \sin \gamma}}\right]^{2}}, \\
\gamma \in\left[13.5^{o}, 90^{\circ}\right] \\
-\frac{v}{c} f_{L 1} \sqrt{1-\left[\frac{r(1-\sin \gamma)+h}{\sqrt{(r+h)^{2}+r^{2}-2 r(r+h) \sin \gamma}}\right]^{2}}, \\
\gamma \in\left[90^{\circ}, 166.5^{\circ}\right]
\end{array}\right.
$$

Since SV travels in a constant speed, $v$, the Doppler shift, $f_{D}$, can be expressed as a function of time as follows:

$$
f_{D}=\left\{\begin{array}{c}
\frac{v}{c} f_{L 1} K, \quad t \in\left[0, \frac{1.3352}{w}\right] \\
-\frac{v}{c} f_{L 1} K, \quad t \in\left[\frac{1.3352}{w}, \frac{2.6704}{w}\right]
\end{array}\right.
$$

where

$$
K=\sqrt{1-\left[\frac{r(1-\sin (w t+0.2356))+h}{\sqrt{(r+h)^{2}+r^{2}-2 r(r+h) \sin (w t+0.2356)}}\right]^{2}}
$$

where $w$ is the angular velocity of SV obtained as $w=\frac{v}{r+h}$. Figure 8 shows the Doppler shift $f_{D}$ as a function of $\gamma$, where it is noticed that, the closer the position of SV to the zenith of the receiver, the smaller the Doppler shift; and, the farther the position of SV to the zenith of the receiver, the larger the Doppler shift.

\section{Appendix 2}

Visible region in the orbital planefor an off -plane GPS receiver

This section investigates the case when the stationary GPS receiver resides on the surface of the earth at a point $R$ which does not lie on the same orbital plane as SV. In this case, the visible arc of the orbit of SV is $\widehat{P Q}$ which together with line $P Q$ encloses the shadowed part of Figure 5a. In Figure 5a, the plane $R P Q$ is the tangent plane to the earth surface passing through $R ; P Q$ is the intersection between the tangent plane $R P Q$ and the orbital plane; $\mathrm{OI} \perp \mathrm{PQ}$ at point $I ; R_{p}$ is the intersection between $O I$ and the earth surface, which is the point intersecting the orbital plane of SV and the shortest arc along the earth surface from $R$ to the orbital plane; $q$ is the shortest distance from the origin $\mathrm{O}$ to $P Q ; \alpha$ is the angle $\angle P O Q$, corresponding to the visible arc $\widehat{P Q}$ and $\beta$ is the elevation angle, $\angle R O I$. From Figure $5 \mathrm{a}$, we have

$$
\begin{aligned}
& \cos \beta=\frac{r}{q}, \quad \text { and } \\
& \cos \frac{\alpha}{2}=\frac{q}{h+r} .
\end{aligned}
$$

Thus, the angle $\alpha$ can be expressed as a function of the elevation angle $\beta$ as

$$
\alpha=2 \cos ^{-1}\left(\frac{r}{(h+r) \cos \beta}\right),
$$

for $|\beta| \leq 76.1^{\circ}$. As shown in Figure $5 \mathrm{~b}$, the maximum value of $\alpha, 153^{\circ}$, occurs when $\beta=0^{\circ}$, i.e. when the receiver lies in the orbital plane of SV; while $\alpha=0^{\circ}$ occurs when $|\beta| \geq 76.1^{\circ}$, i.e. when the receiver cannot see SV on the orbit. It can also be shown that the average value of $\alpha$ is $137^{\circ}$ which occurs when $\beta= \pm 49^{\circ}$.

\section{Endnotes}

${ }^{\text {a The }} \mathrm{C} / \mathrm{A}$ code can mitigate NBI by up to $30 \mathrm{~dB}$ when the $\mathrm{OBI}$ is as usual $1 \mathrm{~ms}$ due to the processing gain. Even when the OBI is increased to its maximum $20 \mathrm{~ms}$, the processing gain only comes up to $43 \mathrm{~dB}$ [21]. When the receiver is in close proximity to the jammer and the NBI is larger than the processing gain, the positioning performance is poor. ${ }^{\mathrm{b}}$ Here, $2.048 \mathrm{MHz}$ instead of 2.046 $\mathrm{MHz}$ is used for calculation convenience. ${ }^{\mathrm{C}}$ If the number of SVs in GPS increases, the number of occupied subcarriers by each SV will get reduced but the designed MC code is similar. ${ }^{d}$ Doppler shift due to SV motion has a negligible effect on the reception of the MC code, which will be analyzed in detail in Section $6 .{ }^{\mathrm{e}}$ Only the mainlobe of the subcarrier is taken into consideration because the majority of signal energy locates in this area. Interfering on the sidelobe by NBI does not affect the probing signal significantly. ${ }^{\mathrm{f}}$ The 'adjacencies' is 
defined as the SV which in the frequency domain are close to " $\mathrm{X}$ " and can possibly collide with " $\mathrm{X}$ ". ${ }^{\mathrm{g}}$ This representation denotes $p_{1}$ (collision $\mid 1$ visible $)=24.3 \%$ when the $\mathrm{OBI}$ is equal to $1 \mathrm{~ms}$ and $p_{1}$ (collision $\mid 1$ visible) $=1.42 \%$ when the OBI is equal to $20 \mathrm{~ms}$. For convenience, we use this representation throughout this article.

\section{Acknowledgements}

This work is supported by the Alberta Informatics Circle of Research Excellence (iCORE), the Natural Sciences and Engineering Research Council of Canada (NSERC), the Canada Research Chair (CRC) Program, TRLabs and the Cell-Loc Location Technologies Inc. Canada.

\section{Author details}

'ECE Department, University of Calgary, Calgary, AB, T2N 1N4, Canada ${ }^{2}$ Green Radio And Signal Processing (GRASP) Group, ECE Department, New York Institute of Technology at Nanjing Campus, China, 210046

\section{Competing interests}

The authors declare that they have no competing interests.

Received: 6 October 2011 Accepted: 31 May 2012

Published: 31 May 2012

\section{References}

1. L Dai, Z Wang, J Wang, Z Yang, Positioning with OFDM signals for the next-generation GNSS. IEEE Trans Consum Electron. 56, 374-379 (2010). doi:10.1109/TCE.2010.5505942

2. J Li, G Liao, O Shan, Joint frequency ambiguity resolution and accurate timing estimation in OFDM systems with multipath fading. EURASIP J Wirel Commun Netw. 2006, 1-7 (2006). doi:10.1155/WCN/2006/62173

3. CN Kishore, VU Reddy, Robust OFDM timing synchronisation in multipath channels. EURASIP J Wirel Commun Netw. 2006, 1-8 (2006). doi:10.1155/ WCN/2006/8614

4. C Williams, S McLaughlin, MA Beach, Robust OFDM timing synchronisation in multipath channels. EURASIP J Wirel Commun Netw. 2008, 1-12 (2008). doi: $10.1155 / 2008 / 675048$

5. L Hanzo, M Munster, B Choi, T Keller, OFDM and MC-CDMA for Broadband Multi-user Communications, WLANs, and Broadcasting, Wiley, West Sussex, (2003)

6. U Tureli, D Kivanc, H Liu, Experimental and analytical studies on a highresolution OFDM carrier frequency offset estimator. IEEE Trans Veh Technol. 50, $2(2001)$

7. U Tureli, H Liu, M Zoltowski, OFDM blind carrier offset estimation: ESPRIT. IEEE Trans Commun. 48, 9 (2000)

8. J Li, M Kavehrad, Effects of time selective multipath fading on OFDM systems for broadband mobile applications. IEEE Commun Lett. 3, 12 (1999)

9. D Wang, M Fattouche, OFDM transmission for time-based range estimation. IEEE Signal Process Lett. 17, 6 (2010)

10. D Wang, M Fattouche, Multipath mitigation for LOS TBRE using NDB OFDM transmission and phase correlation. IET Electron Lett. 46, 21 (2010). doi:10.1049/el.2010.3254

11. F Zanier, M Luise, Fundamental issues in time-delay estimation of multicarrier signals with applications to next-generation GNSS. Proceedings of The 10th Workshop on Signal Processing for Space Communications (SPSC), Rhodes Island 1-8 (Oct. 2008)

12. P Misra, in Global Positioning System: Signals, Measurements, and Performance, 2nd edn. (Ganga-Jamuna Press, Massachusetts, 2006)

13. A Leick, GPS Satellite Surveying 3rd edn. (Wiley, New Jersey, 2004)

14. E Kaplan, C Hegarty, Understanding GPS: Principles and Applications, 2nd edn. Artech House, Boston, (2005)

15. B Parkinson, J Spilker, Global Positioning System: Theory and Applications (American Inst. Aeronautics, Washington DC, 1996)

16. S Kay, Fundamentals of Statistical Signal Processing: Estimation Theory, 2nd edn. (Prentice-Hall, Upper Saddle River, 1998)

17. T Logsdon, The NAVSTAR Global Positioning System Van Nostrand Reinhold, New York, (1992)
18. MS Braasch, AJ Dierendonck, GPS receiver architectures and measurements. Proc IEEE. 87, 1 (1999)

19. M Dinesh, S Yongcheol, S Ryosuke, GPS signal acquisition and tracking an approach towards development of software-based GPS receiver. In Proceedings of The Institute of Electronics, Information and Communication Engineers, Technical Report of IEICE, German (2006)

20. D Manandhar, Y Suh, R Shibasaki, GPS signal acquisition and tracking: an approach towards development of softwarebased GPS receiver. IEICE, Tech Rep ITS2004-16 (Jul 2004)

21. Z Ponos, M Dukic, Analysis of GPS receiver anti-jamming characteristics. IEICE Trans Commun. E83-B, 10 (2000)

22. F Athley, Threshold region performance of maximum likelihood direction of arrival estimators. IEEE Trans Signal Process. 53, 4 (2005)

23. D John, R John, B Robert, R Chris, Manual of Geospatial Science and Technology (CRC Press, Boca Raton, 2002)

doi:10.1186/1687-1499-2012-185

Cite this article as: Wang et al: Multicarrier code for the next-

generation GPS. EURASIP Journal on Wireless Communications and Networking 2012 2012:185.

\section{Submit your manuscript to a SpringerOpen ${ }^{\mathcal{O}}$ journal and benefit from:}

- Convenient online submission

- Rigorous peer review

- Immediate publication on acceptance

- Open access: articles freely available online

- High visibility within the field

- Retaining the copyright to your article

Submit your next manuscript at $\gg$ springeropen.com 